\title{
Theoretical Peak Performance and Optical Constraints for the Deflection of an S-type Asteroid with a Continuous Wave Laser
}

\author{
Nicolas Thiry ${ }^{1}$, Massimiliano Vasile ${ }^{2, *}$ \\ University of Strathclyde, 75 Montrose St, G1 1XJ, Glasgow, UK
}

\begin{abstract}
This paper presents a theoretical model to evaluate the thrust generated by a continuous wave $(\mathrm{CW})$ laser, operating at moderate intensity $\left(<100 \mathrm{GW} / \mathrm{m}^{2}\right)$, ablating an S-type asteroid made of Forsterite. The key metric to assess the performance of the laser system is the thrust coupling coefficient which is given by the ratio between thrust and associated optical power. Three different models are developed in the paper: a one dimensional steady state model, a full 3D steady state model and a one dimensional model accounting for transient effects resulting from the tumbling motion of the asteroid. The results obtained with these models are used to derive key requirements and constraints on the laser system that allow approaching the ideal performance in a realistic case.
\end{abstract}

Keywords: Laser Ablation, Asteroid, Deflection, CW Laser, Planetary Defense, Impulse Coupling

\section{Introduction}

Deflection methods can be divided into two main categories: impulsive and slow-push. Impulsive techniques are generally modelled with an instantaneous change of momentum given by, for example, a nuclear explosion

\footnotetext{
*Corresponding author

Email addresses: nicolas.thiry@strath.ac.uk (Nicolas Thiry), massimiliano.vasile@strath.ac.uk (Massimiliano Vasile)

${ }^{1} \mathrm{PhD}$ Student at the University of Strathclyde

${ }^{2}$ Professor at the University of Strathclyde
} 
(nuclear interceptor) or the hypervelocity impact of a spacecraft (kinetic impactor) with the asteroid. Slow-push methods, on the other hand, allow for a more controllable deflection manoeuvre by exerting a small continuous and controllable force on the asteroid over an extended period of time. The classification of methods in impulsive and slow-push is, therefore, to be considered of operational nature and does not reflect the exact physical interaction between the spacecraft and the asteroid or the physical phenomenon that is generating the change of momentum.

Over the past years many slow-push concepts have been proposed and studied at various degrees of accuracy. Many of them are based on the use of electric propulsion and therefore require a dedicated propulsion system and propellant to generate the necessary deflection. In contrast, slow-push ablation-based methods (such as direct solar or laser ablation) aim at exploiting the material the asteroid is made of, to generate the required thrust. In the work of Kahle et al. (2006) and Vasile and Maddock $(2010)$, however, it was shown that the contamination of the solar collectors, severely limits the effectiveness of direct solar ablation. On the other hand, as demonstrated by Vasile et al. (2014), if the deflection is achievable in a given limit time, laser ablation techniques require a lower mass into space than electric propulsion methods. The use of lasers, compared to directly focusing the light of the Sun, implies higher conversion losses but has the distinctive advantage to provide high light intensity at lower power and longer distance from the target.

Several mission concepts using in-space laser ablation have been investigated in the past using a single laser powered by nuclear reactors (Park and Mazanek (2005); Yoo et al. (2009)) or swarms of lasers powered by the Sun (Vasile and Maddock (2012)). A swarm of spacecraft flying in formation in the proximity of the target asteroid allows combining multiple beams to produce a high thrust level while keeping the power and thermal systems to a manageable size and complexity on each individual spacecraft. In addition, the swarm offers a more reliable system (Zuiani et al. (2012)) in the case of failure of a single spacecraft and a more controllable thrust vector as ablating different parts of the asteroid surface will reduce the uncertainty on the direction of the resultant thrust vector.

Recently a study supported by the European Space Agency, indicated the feasibility of laser ablation at changing the orbital velocity of a 130 tons Stype asteroid by $1 \mathrm{~m} / \mathrm{s}$ in less than 1 year. The concept, called Light Touch ${ }^{2}$ (Vasile et al. (2013)), was considering the use of a commercial fibre laser, 
installed on a $453 \mathrm{~kg}$ spacecraft, and requiring between $460 \mathrm{~W}$ and $860 \mathrm{~W}$ of input power.

For all these scenarios, the performance of laser ablation is dependent on a key parameter: the thrust coupling coefficient. The thrust coupling coefficient is here defined as the ratio between the thrust magnitude and the optical power required to generate that thrust. In this paper, we derive a simple analytical model to estimate the thrust coupling coefficient of a generic Continuous Wave (CW) laser, ablating an S-type asteroid. The model proposed in this paper improves over the one developed by Vasile et al. (2014, 2013) as it more rigorously derives, from conservation laws, the thrust coupling coefficient as a function of material properties and rotation speed of the asteroid. Furthermore, the applicability of the analytical model was checked against the results of higher fidelity numerical models employing the finite element method and the finite volume method to include possible conduction losses perpendicular to the incident laser beam or additional conduction losses due to transient effects induced by the relative motion of the asteroid respectively.

The paper is structured as follows. In section 2, the basic physics of the ablation process is reviewed and the important physical parameters are presented. From this, and taking the assumption of unidimensionality and a steady-state operation regime the analytical model of the thrust coupling is derived in section 3.1. Predictions from this analytical model are then compared to the results of two higher fidelity numerical models respectively including the geometrical effects due to lateral conduction as well as the Gaussian profile of the laser beam in section 4 and the transient effects due to the asteroid rotation in section 5. Through theoretical considerations and by fitting the results of these higher fidelity models, correction laws are derived, allowing the analytical model to provide sufficiently accurate predictions even within the working hypotheses of the higher fidelity numerical models. These results are then used to size the laser system to be installed on board a spacecraft to achieve a given deflection performance in section 6 .

\section{Vaporization of an S-type Asteroid}

In this section we review the basic physics underneath the vaporization process and extract the physical parameters necessary to build the different models presented in the paper. 


\subsection{Energy Balance}

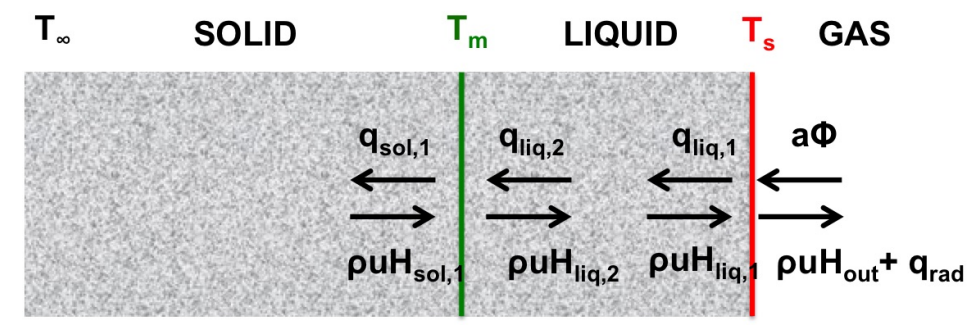

Figure 1: Energy transport during the ablation process

With reference to Figure 1, one can assume that, in general, a layer of asteroid material will undergo a transition from solid to liquid to vapour, when illuminated by the laser. This is consistent with the surface temperature registered during laboratory experiments by Gibbings et al. (2013) and the triple point temperature of the materials most commonly found on S-type asteroids. In each phase, the temperature distribution and evolution will be dictated by the heat equation:

$$
\frac{\partial \rho H}{\partial t}+\nabla \cdot(\rho \mathbf{u} H)=-\boldsymbol{\nabla} \cdot \boldsymbol{q}
$$

In this equation, $H$ is the enthalpy of the material, $\mathbf{u}$ the velocity of the material with respect to the illuminated surface, $\rho$ the density, and $\boldsymbol{q}$ the heat flux. If one considers only the regime state, the time-dependency in Eq. (1) vanishes. By using the Gauss-Ostrogradsky theorem, Eq.(1) can be integrated over the boundary of an arbitrary control volume to give:

$$
\int_{S} \rho \mathbf{u} H \cdot \mathbf{n} d S+\int_{S} \boldsymbol{q} \cdot \mathbf{n} d S=0
$$

In which $\mathbf{n}$ is the unit vector normal to the surface of integration. Thus, in the regime state, the heat conducted through the material is equal to the heat required to heat the vaporized material from its initial state. In the general case, a simple energy balance at the different interfaces (see Figure 1) also yields the following relations:

$$
\begin{aligned}
& q_{l i q, 1}=a \Phi-q_{r a d}-\rho u_{v} H_{v} \\
& q_{l i q, 2}=q_{s o l, 1}+\rho u_{m} H_{m}
\end{aligned}
$$


where $a$ is the absorption coefficient, $\Phi$ is the laser flux, $H_{v}$ and $H_{m}$ are the Enthalpy of vaporization and melting respectively, and $u_{v}$ and $u_{m}$ are the recession speed of the two interfaces, $q_{s o l, 1}, q_{l i q, 1}$, and $q_{l i q, 2}$ the conduction heat fluxes on the appropriate sides of the 2 interfaces, and $q_{\text {rad }}$ the radiated heat flux which can be computed from the Stefan-Boltzmann law: $q_{\text {rad }}=\epsilon \sigma\left(T_{s}^{4}-T_{\infty}^{4}\right)$. In a steady state regime, the 2 recession speeds are identical and equal to $u$ while the internal energy, in any control volume, becomes invariant with respect to time. This means that the heat conducted in the material is balanced by the heat required to increase the temperature, from the rest temperature, of the material escaping the vaporization front. By neglecting the heat conducted in the radial direction and radiated back outwards in the vicinity of the spot, an implicit relation can be found that links directly the recession speed of the interface, its surface temperature $T_{s}$ and the laser flux:

$$
a \Phi=\epsilon \sigma\left(T_{s}^{4}-T_{\infty}^{4}\right)+\rho u\left(H_{v}+H_{m}+c_{l i q}\left(T_{s}-T_{m}\right)+c_{s o l}\left(T_{m}-T_{\infty}\right)\right)
$$

Note that $c_{l i q}$ and $c_{s o l}$ denote the heat capacities in the liquid and solid phases respectively and $\rho$ without index is the density of the condensed material. In the next subsection, we show how the interface velocity u can be expressed as a function of the interface temperature. In section 3, we will show how the particular case covered by Eq. 5 can be used to derive an implicit relation between the laser flux and thrust coupling coefficient, when the 1D steadystate assumptions hold.

\subsection{Knudsen Layer}

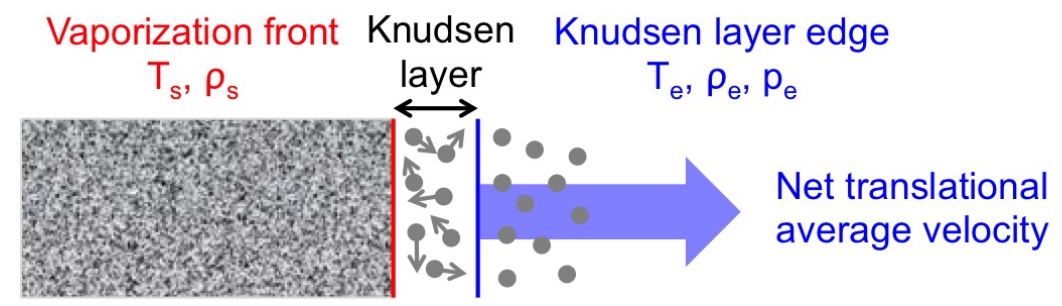

Figure 2: The Knudsen Layer

The conservation of mass across the interface implies a change of velocity proportional to the decrease in density. This velocity builds up through 
collisions at molecular level in a thin layer that can be treated as a gasdynamic discontinuity. The jump conditions across this layer have been derived by Knight (1979) and are given by the following equations:

$$
\begin{aligned}
m & =\sqrt{\frac{\gamma}{2}} M_{e} \\
\frac{T_{e}}{T_{s}} & =\left[\sqrt{1+\pi\left(\frac{\gamma-1}{\gamma+1} \frac{m}{2}\right)^{2}}-\sqrt{\pi} \frac{\gamma-1}{\gamma+1} \frac{m}{2}\right]^{2} \\
\frac{\rho_{e}}{\rho_{s}} & =\sqrt{\frac{T_{s}}{T_{e}}}\left[\left(m^{2}+\frac{1}{2}\right) e^{m^{2}} \operatorname{erfc}(m)-\frac{m}{\sqrt{\pi}}\right]+\frac{1}{2} \frac{T_{s}}{T_{e}}\left[1-\sqrt{\pi} m e^{m^{2}} \operatorname{erfc}(m)\right]
\end{aligned}
$$

In which $\gamma$ is the heat capacity ratio, the indices s and e represent the properties of the gas at the interface and on the edge of the Knudsen layer respectively, and $M_{e}$ represents the local Mach number on the edge of the Knudsen layer which, in general, is dependent on the pressure environment downstream of the ablation front. For expansion in vacuum, $M_{e}$ equates 1 as the ablated flow reaches the sonic limit(Knight (1979)). From the conservation of mass, the mass flow rate per unit area can thus be computed from Eqs. (6) and (7) once the properties of the gas at the interface are known:

$$
\rho u=\rho_{e} \underbrace{\sqrt{\gamma R^{*} T_{e}}}_{\text {speed of sound } c_{e}}
$$

In which $R^{*}$ is the specific gas constant. Note that Eq. (8) is formally equivalent to the Hertz-Knudsen-Langmuir formula in vacuum. In order to close the model, a dependency between the surface temperature $T_{s}$ and density $\rho_{s}$ of the gas is still necessary. An explicit relation can be obtained using the ideal gas law and assuming the liquid at the interface is near thermodynamic equilibrium (Knight (1979)). In the next paragraph, we show the derivation

of the necessary material properties and the relation between the density and temperature in the gas at equilibrium.

\subsection{Thermochemical Relations}

The vaporisation process described in the last two sections requires an appropriate characterization of the target material. We consider in this paper the case of a rocky S-type asteroid mainly composed of magnesium iron 
silicates. The usual choice is to consider the vaporization of Fosterite as it has an unusually high melting temperature among the Olivine family (2171K at 1 atm according to Choudhury et al. (1989)). In a layer of Fosterite, various reactions and sub-reactions occur during the vaporization process. A common assumption made by Ahrens and O'Keefe $(1972)$ and Kahle et al. (2006) is that the following reaction dominates during the vaporisation of Fosterite:

$$
\mathrm{Mg}_{2} \mathrm{SiO}_{4}^{\text {liq }} \longrightarrow 2 \mathrm{MgO}^{\text {gas }}+\mathrm{SiO}^{\text {gas }}+\frac{1}{2} \mathrm{O}_{2}^{\text {gas }}
$$

Following this assumption, most of the useful parameters can be directly retrieved or inferred by the data in Table 1, in which the formation enthalpies, free Gibbs enthalpies, heat capacities and stoichiometric coefficients of the different substances are given. One of the most important material prop-

Table 1: Relevant properties at standard pressure (1 atm) extracted from the NISTJANAF Thermochemical Tables 4

\begin{tabular}{lllll}
\hline Substance $(\mathrm{i})$ & $\mathrm{Mg}_{2} \mathrm{SiO}_{4}{ }^{\text {liq }}$ & $\mathrm{MgO}^{\text {gas }}$ & $\mathrm{SiO}^{\text {gas }}$ & $\mathrm{O}_{2}^{\text {gas }}$ \\
\hline$\Delta_{f}^{3500 K} H^{s t d}\left[\mathrm{~kJ} \mathrm{~mol}^{-1}\right]$ & -2301.5 & -70.154 & -178.375 & 0 \\
$\Delta_{f}^{3000 K} G^{\text {std }}\left[\mathrm{kJ} \mathrm{mol}^{-1}\right]$ & -686.991 & -3.414 & -300.935 & 0 \\
$C_{p}\left[\mathrm{~J} \mathrm{~mol}^{-1} \mathrm{~K}^{-1}\right]$ & 205 & 42 & 38 & 40 \\
$n_{i}$ & - & 2 & 1 & $\frac{1}{2}$ \\
\hline
\end{tabular}

erties, in this respect, is the vaporization enthalpy per unit mass which can be computed by simply subtracting the formation enthalpy of the reactant with the formation enthalpy of the products as follows:

$$
\begin{aligned}
H_{v} & =2 \Delta_{f} H_{\mathrm{MgO}^{\text {gas }}}^{s t d}+\Delta_{f} H_{\mathrm{SiO}^{\text {gas }}}^{s t d}+\frac{1}{2} \Delta_{f} H_{\mathrm{O}_{2}{ }^{\text {gas }}}^{\text {std }}-\Delta_{f} H_{\mathrm{Mg}_{2} \mathrm{SiO}_{4}{ }^{\text {liq }}}^{\text {std }} \\
& =0.5665 \mathrm{MJ} \cdot \mathrm{mol}^{-1}=14.163 \mathrm{MJ} \cdot \mathrm{kg}^{-1}
\end{aligned}
$$

The properties of the gas mixture formed during the ablation process can also be retrieved in a similar fashion. They are simply computed by summing the properties of the different compounds weighted by their respective molar or

\footnotetext{
${ }^{4}$ http://kinetics.nist.gov/janaf/
} 
mass fraction in the mixture. The resulting heat capacity per unit mass of the gas mixture $c_{p}^{g}$ is computed from the molar heat capacities $C_{p}$ :

$$
c_{p}^{g a s}=\frac{\sum_{i} n_{i} C_{p}^{i}}{0.14}=1014.3 \mathrm{~J} \cdot \mathrm{kg}^{-1} \cdot \mathrm{K}^{-1}
$$

with a value for the specific gas constant given by:

$$
R^{*}=\frac{\sum_{i} n_{i} R}{0.14}=207.75 \mathrm{~J} \cdot \mathrm{kg}^{-1} \cdot \mathrm{K}^{-1}
$$

Given Eqs. (11) and (12) one can compute the heat capacity ratio $\gamma$ :

$$
\gamma=\frac{c_{p}^{\text {gas }}}{c_{p}^{\text {gas }}-R^{*}}=1.26
$$

In order to compute the properties of the gas at the interface, we assume that the liquid at the interface is near thermodynamic equilibrium, which is a reasonable assumption for fluxes below $100 \mathrm{GW} / \mathrm{m}^{2}$ according to Knight (1979). A pressure-temperature dependency can thus be sought by studying the phase transition of Forsterite through the prism of thermodynamics. Under thermodynamic equilibrium the chemical potentials $\mu$ are equal in all phases. Thus, for such an equilibrium state $\left\{p_{r e f}, T_{r e f}\right\}$, that we will take as reference state, the following relation holds:

$$
\Delta^{\text {gas-liq }} \mu\left(p_{r e f}, T_{r e f}\right)=\sum_{i} n_{i} \mu_{i}^{g a s}\left(p_{r e f}^{i}, T_{r e f}\right)-\mu^{l i q}\left(p_{r e f}, T_{r e f}\right)=0
$$

From this reference state, one can compute the evolution of the chemical potentials with pressure and temperature. Keeping the pressure constant, the variation between two different temperature states can be derived from the Gibbs-Helmholtz equation:

$$
\frac{\Delta^{\text {gas-liq }} \mu\left(p_{r e f}, T_{s}\right)}{T_{s}}-\frac{\Delta^{\text {gas-liq }} \mu\left(p_{r e f}, T_{r e f}\right)}{T_{r e f}}=H_{v}^{\text {mol }}\left(\frac{1}{T_{s}}-\frac{1}{T_{r e f}}\right)
$$


On the other hand, if the temperature is maintained constant, the pressure dependency in the gas mixture is computed knowing that:

$$
\begin{aligned}
\frac{n_{i}}{\sum_{i} n_{i}} & =\frac{p_{s}^{i}}{p_{s}}=\frac{p_{\text {ref }}^{i}}{p_{\text {ref }}} \Rightarrow \frac{p_{s}^{i}}{p_{\text {ref }}^{i}}=\frac{p_{s}}{p_{\text {ref }}} \\
\mu_{i}^{g a s}\left(p^{i}, T_{s}\right) & =\mu_{i}^{g a s}\left(p_{\text {ref }}^{i}, T_{s}\right)+R T_{s} \ln \left(\frac{p^{i}}{p_{\text {ref }}^{i}}\right) \\
& =\mu_{i}^{\text {gas }}\left(p_{\text {ref }}^{i}, T_{s}\right)+R T_{s} \ln \left(\frac{p_{s}}{p_{\text {ref }}}\right)
\end{aligned}
$$

Where $p_{\text {ref }}^{i}$ and $p_{\text {ref }}^{i}$ are the partial pressures at the reference and in the new pressure state respectively. Since the Gibbs free energy is a state variable, one can use these two relations to compute any new state $\left\{p_{s}, T_{s}\right\}$ from the initial state $\left\{p_{r e f}, T_{r e f}\right\}$. By combining both relations one gets the new state:

$$
\frac{1}{T_{s}}\left(\sum_{i} n_{i}\left(\mu_{i}^{g a s}\left(p_{s}^{i}, T_{s}\right)-R T_{s} \ln \left(\frac{p_{s}}{p_{r e f}}\right)\right)-\mu^{l i q}\left(p_{s}, T_{s}\right)\right)=H_{v}^{\text {mol }}\left(\frac{1}{T_{s}}-\frac{1}{T_{r e f}}\right)
$$

For this state to remain an equilibrium, the following must also hold:

$$
\mu^{l i q}\left(p_{s}, T_{s}\right)=\sum_{i} n_{i} \mu_{i}^{g a s}\left(p_{s}^{i}, T_{s}\right)
$$

and by combining Eqs. (17) and (18) one can derive the Clausius-Clapeyron formula for the gas mixture:

$$
\ln \left(\frac{p_{s}}{p_{\text {ref }}}\right)=\frac{H_{v}^{\text {mol }}}{R \sum_{i} n_{i}}\left(\frac{1}{T_{\text {ref }}}-\frac{1}{T_{s}}\right)=\frac{H_{v}}{R^{*}}\left(\frac{1}{T_{\text {ref }}}-\frac{1}{T_{s}}\right)
$$

An assumption in this formula is that the vaporization enthalpy doesn't vary too much with the temperature, which is true for limited temperature extrapolations. The pressure of the reference state can be computed from the formation free Gibbs enthalpy of the different species at the reference temperature of $3000 \mathrm{~K}$ in Table 1 .

$$
\begin{aligned}
\Delta G_{\text {mol (gas })}^{\text {std }} & =\sum_{i} \frac{n_{i}}{\sum_{i} n_{i}}\left(\Delta_{f} G_{i}^{s t d}+R T_{\text {ref }} \ln \left(\frac{n_{i}}{\sum_{i} n_{i}}\right)\right)-\frac{\Delta_{f} G_{\text {fost }}^{\text {std }}}{\sum_{i} n_{i}} \\
\Delta G_{\text {mol (gas })}^{\text {ref }} & =\Delta G_{\text {mol }(\text { gas })}^{\text {std }}+R T_{\text {ref }} \ln \left(\frac{p_{\text {ref }}}{10^{5}}\right)=0 \\
p_{\text {ref }} & =10^{5} \exp \left(\frac{-\Delta G_{\text {mol }}^{\text {std }}}{R T_{\text {ref }}}\right)=4448.9 P a
\end{aligned}
$$




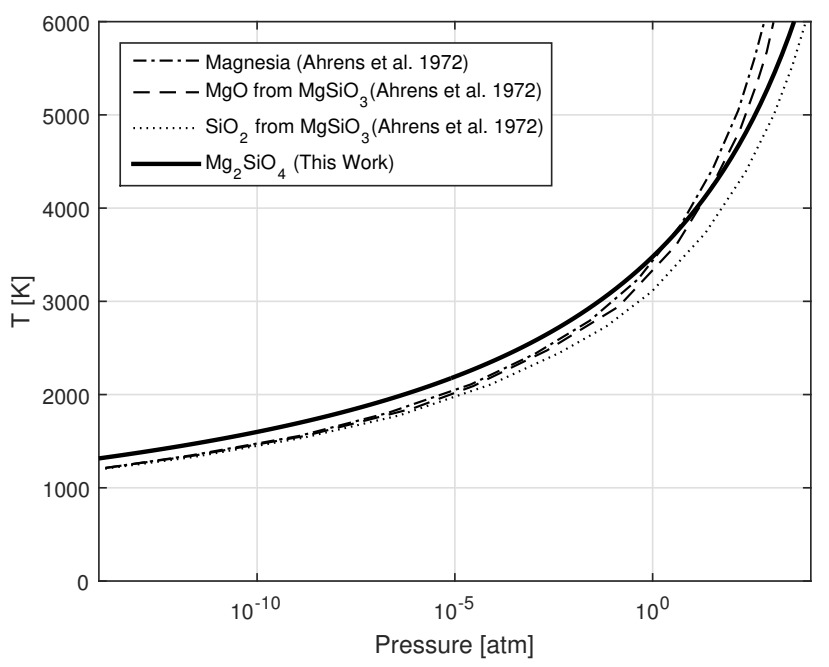

Figure 3: Calculated equilibrium vapor pressure of the system $\mathrm{Mg}_{2} \mathrm{SiO}_{4}{ }^{\text {liq }} \leftrightarrow 2 \mathrm{MgO}^{\text {gas }}+$ $\mathrm{SiO}^{\text {gas }}+\frac{1}{2} \mathrm{O}_{2}{ }^{\text {gas }}$

With this, the whole $p_{s}-T_{s}$ curve can be computed and the result is represented in Figure 3. Note that this curve is consistent with the results of Ahrens and O'Keefe (1972). An explicit relation between $\rho_{s}$ and $T_{s}$ can now be computed if one considers the ideal gas law:

$$
\rho_{s}=\frac{p_{r e f}}{R^{*} T_{s}} \exp \left(\frac{H_{v}}{R^{*}}\left(\frac{1}{T_{r e f}}-\frac{1}{T_{s}}\right)\right)
$$

As a summary of this section, Table 2 shows the different properties considered in the models developed in the remainder of the paper. 
Table 2: Physical properties of Forsterite

\begin{tabular}{lll}
\hline Quantity & Symbol & Value \\
\hline Density & $\rho$ & $3280 \mathrm{~kg} / \mathrm{m}^{3}$ \\
Thermal Conductivity & $k$ & $2 \mathrm{~W} \cdot \mathrm{m}^{-1} \cdot \mathrm{K}^{-1}$ \\
Heat Capacity (liquid) & $c_{l i q}$ & $1464 \mathrm{~J} \cdot \mathrm{kg}^{-1} \cdot \mathrm{K}^{-1}$ \\
Heat Capacity (solid) & $c_{s o l}$ & $1264 \mathrm{~J} \cdot \mathrm{kg}^{-1} \cdot \mathrm{K}^{-1}$ \\
Vaporization Enthalpy & $H_{v}$ & $14.163 \mathrm{MJ} / \mathrm{kg}$ \\
Melting Enthalpy & $H_{m}$ & $0.508 \mathrm{MJ} / \mathrm{kg}$ \\
reference temperature & $T_{r e f}$ & $3000 \mathrm{~K}$ \\
ref. saturation pressure & $p_{r e f}$ & $4448.9 \mathrm{pa}$ \\
Melting point & $T_{m}$ & $2171 \mathrm{~K}$ \\
Gas Constant & $\mathrm{R}^{*}$ & $206.7 \mathrm{~J} \cdot \mathrm{kg}^{-1} \cdot \mathrm{K}^{-1}$ \\
Heat ratio (gas) & $\gamma$ & 1.26 \\
Emissivity & $\epsilon$ & 0.9 \\
Absorptivity & $a$ & 0.8 \\
Rest temperature & $T_{\infty}$ & $298 \mathrm{~K}$ \\
\hline
\end{tabular}




\section{Thrust Coupling Coefficient in the Steady-State Regime}

Based on the physics of the ablation process derived in section 2, in this section we first introduce the thrust coupling coefficient (subsection 3.1), then we derive an estimation for the thickness of the heated layer and finally, in subsection 3.3, we derive an estimation of the time required to reach steady state conditions. In sections 4 and 5, the model proposed in this section will be compared to higher fidelity numerical models accounting for a full 3D geometry and non-steady state conditions. This will allow us to understand which factors limit the ideal performances predicted by the present model and, subsequently, to derive design constraints on the laser system that mitigate these factors.

\subsection{Thrust Coupling Coefficient}

One of the key figures of merit of laser-based deflection methods is the thrust coupling coefficient $C_{m}$. The thrust coupling coefficient is defined as the ratio of the force generated by a given incident optical power and the optical power itself. It can also be computed as the ratio between the effective force per unit area $p_{\text {eff }}$ and the optical flux $\Phi$ :

$$
C_{m}=\frac{p_{e f f}}{\Phi}
$$

The effective force per unit area can be computed by summing up the rate of change of momentum to the pressure at the edge of the Knudsen layer:

$$
\begin{aligned}
p_{e f f} & =p_{e}+\rho_{e} c_{e}^{2} \\
& =(\gamma+1) p_{e}
\end{aligned}
$$

Where $c_{e}$ is the local speed of sound in the gas. From the ideal gas law, the pressure at the edge of the Knudsen layer, $p_{e}$, can also be derived from Eq. (21) as a function of the temperature $T_{s}$ only:

$$
p_{e}=\rho_{e} R^{*} T_{e}=p_{\text {ref }}\left(\frac{\rho_{e}}{\rho_{s}} \frac{T_{e}}{T_{s}}\right) \exp \left(\frac{H_{v}}{R^{*}}\left(\frac{1}{T_{r e f}}-\frac{1}{T_{s}}\right)\right)
$$

since the ratios of temperatures and densities are function of the heat capacity ratio only according to Eqs. (6) and (7). Then, by combining Eqs. (6), 7 , 8. 21) into Eq. (5) one can also see that the surface temperature $T_{s}$ is a function of the laser flux only. 
It follows that, in the regime state, the thrust coupling coefficient depends only on the laser flux on the target. Figure 4 shows the estimated thrust coupling coefficient and the surface temperature as a function of the flux. For intensities inferior to $1 \mathrm{MW} / \mathrm{m}^{2}$, the surface temperature is not sufficient to produce a significant level of vaporisation. On the other hand, laser fluxes beyond $100 \mathrm{MW} / \mathrm{m}^{2}$ appear to be sufficient to deliver a thrust higher than $60 \mu N$ per watt of optical power. Note that this value is in line with the recent experimental results of Brashears et al. (2015), who measured the thrust coupling coefficient of similar materials with a $\mathrm{CW}$ laser.

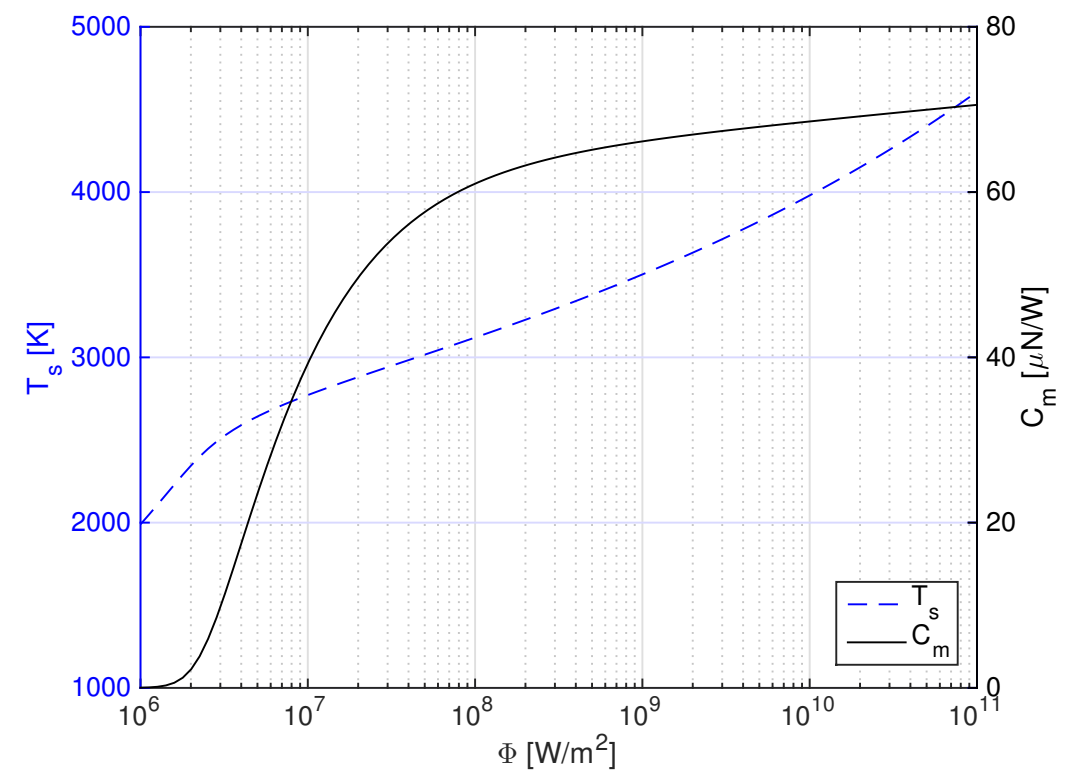

Figure 4: Predicted thrust coupling and surface temperature for a range of possible laser fluxes

\subsection{Thickness of the heated layer in the steady-state regime}

One key assumption underneath the result in Figure 4 is that the thermal gradient is perpendicular to the illuminated surface and dissipation in lateral directions is negligible (1D assumption). This assumption is equivalent to saying that the thickness of the heated layer is small compared to the diameter of the laser beam. It is, therefore, important to compute an estimation 
of this thickness to check when this $1 \mathrm{D}$ assumption holds for a given laser system.

The temperature distribution as a function of the depth $z$ can be computed by solving the 1D advection-diffusion problem derived from the general equation (1). For the sake of simplicity, we consider here identical material properties in the molten and solid phases. As this assumption is not necessary for the analytical model of the previous section, it is only used here to derive a simple expression of the temperature profile while the actual heat capacities of the solid and molten phases actually vary by about $16 \%$ if one refers to table 2. Considering this, the $1 \mathrm{D}$ advection-diffusion equation reads:

$$
u \frac{d T}{d z}+\alpha \frac{d^{2} T}{d z^{2}}=0
$$

where $\alpha=\frac{k}{\rho c}$ is the thermal diffusivity. The generic solution is in the form $T(z)=A \exp \left(-\frac{u}{\alpha} z\right)+B$. The temperature profiles can be computed both in the molten and solid phases by setting the following boundary conditions:

$$
\begin{aligned}
T(z=0) & =T_{s} \\
T\left(z=z_{m}\right) & =T_{m} \\
T(z \rightarrow \infty) & =T_{\infty}
\end{aligned}
$$

The resulting temperature distribution through the condensed phases is then given by:

$$
T(z)= \begin{cases}\frac{T_{s}-T_{m}}{1-\exp \left(-\frac{u}{\alpha} z_{m}\right)} \exp \left(-\frac{u}{\alpha} z\right)+\frac{T_{m}-\exp \left(-\frac{u}{\alpha} z_{m}\right) T_{s}}{1-\exp \left(-\frac{u}{\alpha} z_{m}\right)} & \text { if } z<z_{m} \\ \left(T_{m}-T_{\infty}\right) \exp \left(-\frac{u}{\alpha}\left(z-z_{m}\right)\right)+T_{\infty} & \text { if } z_{m}<z\end{cases}
$$

The location of the melting front is obtained by using the heat balance at the melting interface:

$$
\frac{k u}{\alpha} \frac{T_{s}-T_{m}}{\exp \left(\frac{u}{\alpha} z_{m}\right)-1}=\frac{k u}{\alpha}\left(T_{m}-T_{\infty}\right)+\rho u H_{m}
$$

and by rearranging the terms of Eq. (30), we find:

$$
z_{m}=\frac{\alpha}{u} \log \left(\frac{T_{s}-T_{m}}{T_{m}-T_{\infty}+\frac{H_{m}}{c}}+1\right)
$$


Figure 5 shows the resulting temperature distribution in the asteroid material according to Eq. (29) if the laser flux is such that a temperature of $3000 \mathrm{~K}$ is reached at the vaporization front. The value $l_{c}$ is defined as $l_{c}=\alpha / u$ and would correspond to the intersection of the slope of the temperature profile at $z=0$ with the horizontal axis $T=T_{\infty}$ if $H_{m}$ was equal to 0 .

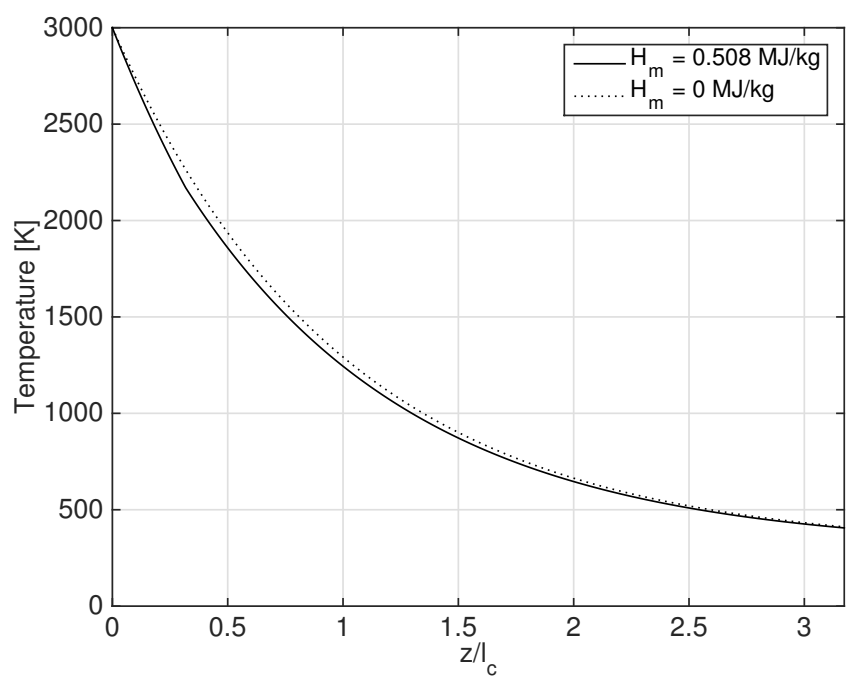

Figure 5: Temperature distribution under the laser spot as a function of the normalized depth for different values of the melting enthalpy $\mathrm{H}_{m}$

The exponential temperature distribution in figure 5 shows that the layer of material that is heated from a temperature close to the rest temperature $T_{\infty}$ to the surface temperature $T_{s}$ is indeed proportional to $l_{c}$. The thermal gradient in the axial direction is therefore proportional to $\frac{T_{s}-T_{\infty}}{l_{c}}$ while the diameter of the laser beam $D_{b}$ drives the importance of the thermal gradients in the lateral directions. As a consequence, one would reasonably expect that the 1D assumption is justified whenever the ratio $\frac{D_{b}}{l_{c}}$ is high enough. In section 4, we demonstrate the validity of this theoretical result and quantify the meaning of "high enough".

\subsection{Time to reach the steady-state regime}

Another important assumption is that the vaporisation process proceeds in steady-state conditions. For this assumption to hold true, the material 
needs to be exposed to the laser beam for enough time to reach the steadystate regime, hence an estimation of this time is required. The time to reach a steady-state $\tau_{c}$ can be estimated by considering the $1 \mathrm{D}$ transient heat equation:

$$
\frac{\partial T}{\partial t}=u \frac{\partial T}{\partial z}+\alpha \frac{\partial^{2} T}{\partial z^{2}}
$$

Choosing a set of non-dimensional variables such as $t=\tau_{c} \hat{t}, z=l_{c} \hat{z}, T=$ $\left(T_{s}-T_{\infty}\right) \hat{T}$, Eq. 32 can be rewritten as:

$$
\frac{\partial \hat{T}}{\partial \hat{t}}=\frac{u^{2} \tau_{c}}{\alpha}\left(\frac{\partial \hat{T}}{\partial \hat{z}}+\frac{\partial^{2} \hat{T}}{\partial \hat{z}^{2}}\right)
$$

By using a simple dimensional analysis, one can see that $\tau_{c}$ is given by the proportionality relationship:

$$
\tau_{c} \propto \frac{\alpha}{u^{2}}
$$

From Eq. (5), one can also note that the interface speed can be crudely approximated by the following ratio:

$$
u \approx \frac{a \Phi}{\rho H_{v}}
$$

Therefore, the characteristic time itself must be:

$$
\tau_{c} \propto\left(\frac{\Gamma}{a \Phi} \frac{H_{v}}{c}\right)^{2}
$$

where $\Gamma=\sqrt{\rho c k}$ is the thermal inertia of the asteroid. A similar expression can be derived by considering the heat equation without vaporization:

$$
\frac{\partial T}{\partial t}=\alpha \frac{\partial^{2} T}{\partial z^{2}}
$$

with boundary condition:

$$
-k \frac{\partial T}{\partial z}=a \Phi \text { in } \mathrm{z}=0
$$

The analytical solution of Eq. (37) can be found in reference textbooks Anisimov and Khokhlov (1995)):

$$
T(z, t)=\frac{2 a \Phi}{k} \sqrt{\alpha t} \operatorname{ierfc}\left(\frac{z}{2 \sqrt{\alpha t}}\right)+T_{\infty}
$$


This shows that, initially, the surface temperature and the heated layer both grow as $\sqrt{\alpha t}$. Setting $z=0$, one can invert this relation to obtain another estimation of $\tau_{c}$ by computing the time required to reach a given surface temperature $T_{s}$ in the absence of vaporisation:

$$
\tau_{c} \propto\left(\frac{\Gamma}{a \Phi} \Delta T\right)^{2}
$$

Interestingly, we notice that both Eqs. (36) and (40) agree that the time to reach a steady-state regime varies according to $\Phi^{-2}$ which is of importance to explain the later analysis carried in this paper.

\subsection{Plasma Ignition Threshold}

The assumption in our model is that operations are carried out at a sufficiently low intensity level so that ionization losses can be neglected. When plasma dominates the laser produced plume, increasing the laser intensity yields a reduction of the value of the coupling coefficient. According to Phipps et al. (1996), in this regime, the dependence between the energy flux and the thrust coupling coefficient follows a power law of the form $C_{m} \propto(\Phi \lambda \sqrt{\tau})^{-1 / 4}$. In the last expression, $\tau$ is the pulse duration and $\lambda$ the laser wavelength. Before this decrease occurs, the $\mathrm{C}_{m}$ typically stalls to a quasi-plateau value for a range of laser intensities.

The processes leading to plasma formation under continuous wave laser irradiation have been investigated by Poueyo-Verwaerde et al. (1993). Initially, an energy flux on the order of $100 \mathrm{GW} / \mathrm{m}^{2}$ is required to accelerate the free electrons in the vapor by inverse Bremsstrahlung (IB) until their kinetic energy becomes sufficient to ionize the atoms of the vapor by an avalanche process. Indeed, as explained by Poueyo-Verwaerde et al. (1993), a necessary condition for the development of this electron avalanche is that the growth rate of electron energy by IB is higher than the losses due to elastic collisions with neutral atoms in the plume. This condition translates in a CW intensity threshold approximately equal to:

$$
\Phi_{\text {plasma }}^{C W}\left(T W / m^{2}\right) \approx \frac{6 U_{i}(e V)}{\lambda^{2}(\mu m) A}
$$

In which $U_{i}$ is the ionization potential and $A$ the atomic mass number. According to this expression, for laser frequencies in the infrared and typical 
values of ionization potentials and atomic masses, the plasma ignition threshold is at intensities ranging between $10 \mathrm{GW} / \mathrm{m}^{2}$ and $1 \mathrm{TW} / \mathrm{m}^{2}$.

For pulsed lasers, an empirical value of the plasma ignition threshold was found by Phipps et al. (1988) as

$$
\Phi_{\text {plasma }}^{\text {pulsed }} \sqrt{\tau}=4.8 \mathrm{E}+08 W m^{-2} s^{1 / 2}
$$

The time-dependency of Eq. 42 might give the impression that in the CW case ionization can occur at relatively low intensities. However, the model treated by Phipps et al. (1996) assumes that absorption of the laser intensity by the plume is done in a time short enough that its 3-dimensional expansion can be neglected. In the case of interest both the expansion and absorption happen simultaneously. Typically, the length of the zone where laser heating dominates over the expansion has a characteristic dimension on the order of the beam spot diameter. To understand which value to give to $\tau$ in Eq. 42 in the case of a CW laser, one must thus estimate the time it takes for the plume to cross such a distance. With a typical ejection speed of $1 \mathrm{~km} / \mathrm{s}$ and beam diameters ranging from 1 to $30 \mathrm{~mm}$, one obtains with the above formula threshold intensities ranging from $88 \mathrm{GW} / \mathrm{m}^{2}$ to $480 \mathrm{GW} / \mathrm{m}^{2}$, which is in good agreement with Eq. 41.

Last but not least, we also implemented a model of the plasma breakdown taking into account the IB absorption by a cloud of vapor with an initial density and internal energy predicted by our equilibrium model. Once vaporized, this cloud will absorb part of the laser light through IB. The evolution of its internal energy is driven by

$$
\frac{d(\rho e)}{d t}=\alpha_{I B} \Phi
$$

In which $\alpha_{I B}$ is the inverse Bremsstrahlung coefficient which can be found in reference textbooks (Vertes et al. (1993)):

$$
\alpha_{I B}\left(m^{-1}\right)=\frac{2 \pi}{3 \mathrm{E}+23}\left(\frac{2 \pi}{3 k_{B} T}\right)^{1 / 2}(2 \pi)^{3} Z^{2} n_{e} n_{i} g_{f f} e^{6} c^{5} \frac{1-\exp \left(-\frac{\hbar \omega}{k_{B} T}\right)}{h m_{e}^{3 / 2} \omega^{3}}
$$

Where $k_{B}$ is the Boltzmann constant, e the electron charge, c the speed of light in vacuum, h the Planck constant, $g_{f f}$ the Gaunt factor, $\omega$ the laser angular frequency, $m_{e}$ the electron mass and $\mathrm{Z}$ the average ionization stage. If we restrict our consideration to a single-stage ionization process, $\mathrm{Z}=1$ and 
$n_{e}=n_{i}=\eta n_{T O T}$, where $\mathrm{n}_{T O T}$ is the number density of atoms in the vapor cloud and $\eta$ is the ion fraction. The latter can be computed considering the Saha equation with the customary simplifications (Vertes et al. (1989)):

$$
\frac{\eta^{2}}{1-\eta}=\frac{1}{n_{T O T}}\left(\frac{2 \pi k_{B} T m_{e}}{h^{2}}\right)^{3 / 2} \exp \left(-\frac{U_{i}}{k_{B} T}\right)
$$

The internal energy of an ideal plasma is given by

$$
\rho e=n_{T O T}\left[\frac{3}{2}(1+\eta) k_{B} T+\eta U_{i}\right]
$$

Eqs. 43, 44, 45, and 46 form a closed system which can be dynamically integrated to find the ion fraction as a function of time. A typical result for aluminium is given on Figure 7, where the calculated intensity to reach an ionization fraction of $5 \%$ is plotted as a function of laser intensity and pulse duration. Our results agree qualitatively with the theory of Phipps et al.

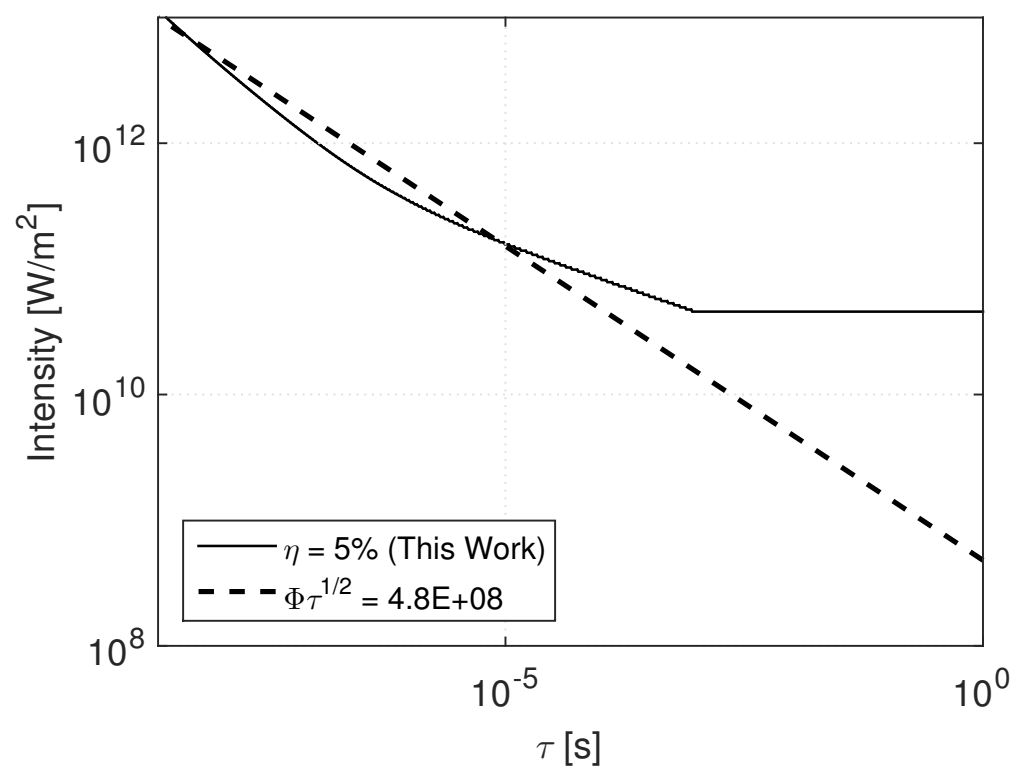

Figure 6: Plasma ignition threshold as a function of the intensity $\Phi$ and duration $\tau$ for aluminium and $\lambda=10.6 \mu \mathrm{m}$ 


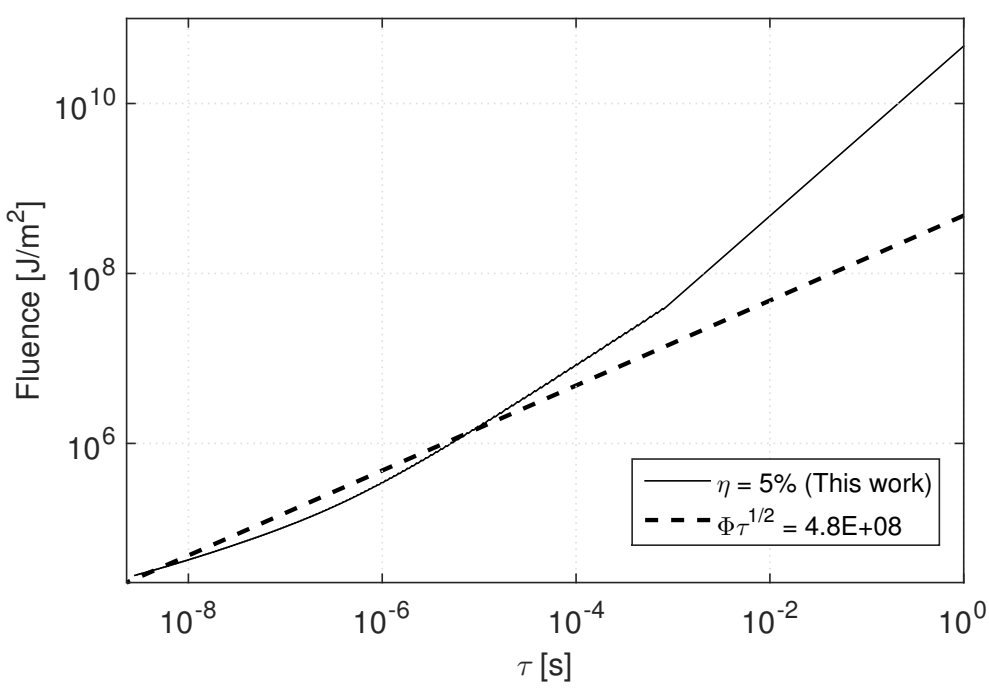

Figure 7: Plasma ignition threshold as a function of the fluence and duration $\tau$ for aluminium and $\lambda=10.6 \mu \mathrm{m}$

(1988) for short pulses up to a time of approximatively 1 millisecond 5 , beyond which significant deviation occurs as 3D expansion becomes the limiting factor for plasma ignition and the ignition threshold is only dependent on laser wavelength and intensity as predicted by the model of Poueyo-Verwaerde et al. (1993). The intensity threshold for plasma ignition found by our model is around $50 \mathrm{GW} / \mathrm{m}^{2}$ at a $10.6 \mu \mathrm{m}$ wavelength for aluminium, which is again in agreement with the values that we derived based on works of PoueyoVerwaerde et al. (1993) and Phipps et al. (1988).

In this paper, we are interested in the lower part of the intensity range where $C_{m}$ is near a plateau. While higher intensities can yield a slight additional increase in performances, our choice is a trade-off between high thrust coupling coefficient and practical requirements on the optics for a CW laser system. In the rest of this paper, we can therefore consider that plasma does not affect the results in the range of intensities of interest, while plotted results for intensities beyond $10 \mathrm{GW} / \mathrm{m}^{2}$ should be considered with caution.

\footnotetext{
${ }^{5}$ Interestingly this time corresponds to the limit of validity the aforementioned author mentions in his work
} 


\section{Losses Due to 3D Effects}

The claim in section 3.2 was that a $1 \mathrm{D}$ approach is justified when the thickness of the heated layer is small in comparison to the size of the laser beam. Indeed, in this case, the thermal gradients in the axial direction will be the dominant terms in the diffusive heat transfer processes. However, the dimensional analysis does not account for non-linear effects caused by the radiation losses in the direct neighbourhood of the laser spot. Moreover, due to the Gaussian intensity profile, deviations from the 1D approach can also occur because part of the energy reaches the target too far from the centercore of the beam and, therefore, can not contribute to the ablation process. In this section, we develop a 3D Finite Element Model (FEM) in order to verify the impact on $C_{m}$ of the unmodelled components in the $1 \mathrm{D}$ model. From the comparison between the higher fidelity 3D model and the 1D model a correction factor is derived that accounts for conduction losses in directions parallel to the surface. Last but not least, the FEM is also used to assess the impact of the transparency of the asteroid material at the wavelength of the laser beam.

\subsection{FEM Axis-Symmetrical Model}

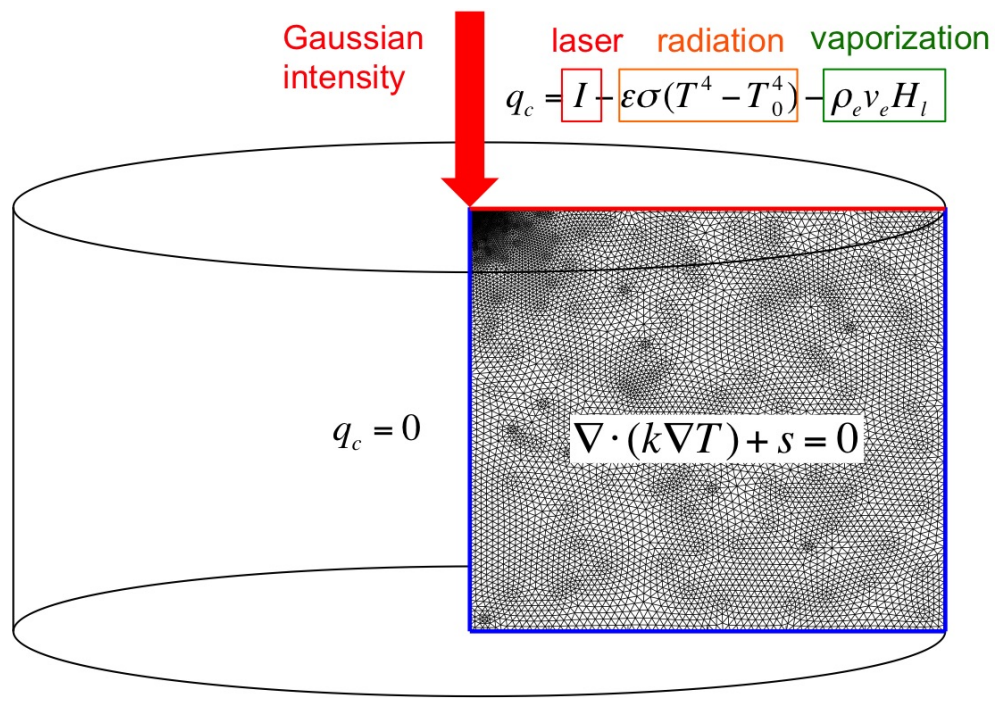

Figure 8: 3D Axis-Symmetrical Model showing mesh and BCs 
The model depicted in figure 8 was implemented in Matlab ${ }^{\circledR}$ taking advantage of the PDE toolbox to solve the heat Eq. (47) in cylindrical coordinates. In a frame attached to the target, this equation writes:

$$
\boldsymbol{\nabla} \cdot(k \boldsymbol{\nabla} T)+s=0
$$

In this equation, $s$ represents a volumic source term which is equal to zero if we assume that the asteroid material is opaque and the power from the laser beam is absorbed at the surface of the ablation spot. However, in the case of a partially transparent material, this term will account for the absorption of the beam intensity through the depth of the material. A typical mesh can be seen on figure 8. The mesh criterion and geometry size is automatically adapted in function of the user-defined output power and diameter of the laser beam. These were initially adjusted by following a thorough sensitivity study to ensure they do not compromise the quality of the results. The boundary conditions are imposed as follow:

- Along the symmetry axis and far region:

$$
q_{c}=0
$$

- On the top surface:

$$
q_{c}=a \Phi-\epsilon \sigma\left(T_{s}^{4}-T_{\infty}^{4}\right)-\rho u H_{l}
$$

In Eq. (49), $H_{l}$ represents an augmented enthalpy which accounts for the total energy required to heat the flow of material leaving the asteroid.

$$
H_{l}=H_{v}+H_{m}+c_{s o l}\left(T_{m}-T_{\infty}\right)+c_{l i q}\left(T_{s}-T_{m}\right)
$$

The main transverse mode of the laser beam (TEM00) is accounted in the model by inputting a Gaussian intensity distribution:

$$
I=a \Phi=\frac{8 a P}{\pi D_{b}^{2}} \exp \left(\frac{-8 r^{2}}{D_{b}^{2}}\right)
$$

In this expression, $D_{b}$ represented the diameter of the laser beam defined classically as four times the standard deviation of the TEM00 mode. Far from the center of the laser beam, this intensity vanishes together with the 
vaporization process so that Eq. (49) naturally tends to the simple radiative boundary conditions:

$$
q_{c}=-\epsilon \sigma\left(T_{s}^{4}-T_{\infty}^{4}\right)
$$

By comparison to the analytical model, the radiations on the sides of the laser spot will now represent an additional energy drain. A non-linear solver is used to find the temperature distribution that is consistent with the heatequation and the non-linear boundary conditions.

Last but not least, the FEM model can also account for a partial transparency of the asteroid material. Knowing the attenuation coefficient $\chi_{a t t}$, the Beer-Lambert law of absorption expresses the remaining intensity I at a given depth of the material.

$$
\frac{d I}{d z}=-\chi_{a t t} a \Phi(r) \exp \left(-\chi_{a t t} z\right)
$$

In which $\Phi(r)$ is given by equation (51). An absorption length $l_{a b s}$ can be defined from this law and is simply equal to the inverse of the attenuation coefficient:

$$
l_{a b s}=\chi_{a t t}^{-1}
$$

The Beer-Lambert law is incorporated in the model by making the following changes:

- Source term:

$$
s(r, z)=-\frac{d I}{d z}=\chi_{a t t} a \Phi(r) \exp \left(-\chi_{a t t} z\right)
$$

- Top boundary conditions:

$$
q_{c}=-\epsilon \sigma\left(T_{s}^{4}-T_{\infty}^{4}\right)-\rho u H_{l}
$$

\subsection{Effect of Lateral Dissipation on the Thrust Coupling Coefficient}

The FEM model provides the temperature profile on the surface and inside the computation domain. From the surface temperature, one can

obtain the net thrust by calculating the following integral over the spot area:

$$
\begin{aligned}
F & =\int_{\text {spot }} p_{e}+\rho_{e} v_{e}^{2} d S \\
& =\int_{\text {spot }}(1+\gamma) p_{e} d S
\end{aligned}
$$


The thrust coupling coefficient is now computed by dividing $F$ by the optical power of the laser $P$ :

$$
C_{m}=\frac{F}{P}
$$

The thrust coupling coefficient was computed for three different power outputs, $0.1 \mathrm{~kW}, 1 \mathrm{~kW}$ and $10 \mathrm{~kW}$, and a spot diameter ranging from 1 to $32 \mathrm{~mm}$. Figure 9 shows that, for a given amount of power, reducing the spot size increases the momentum transferred to the asteroid, as also predicted by the $1 \mathrm{D}$ model.

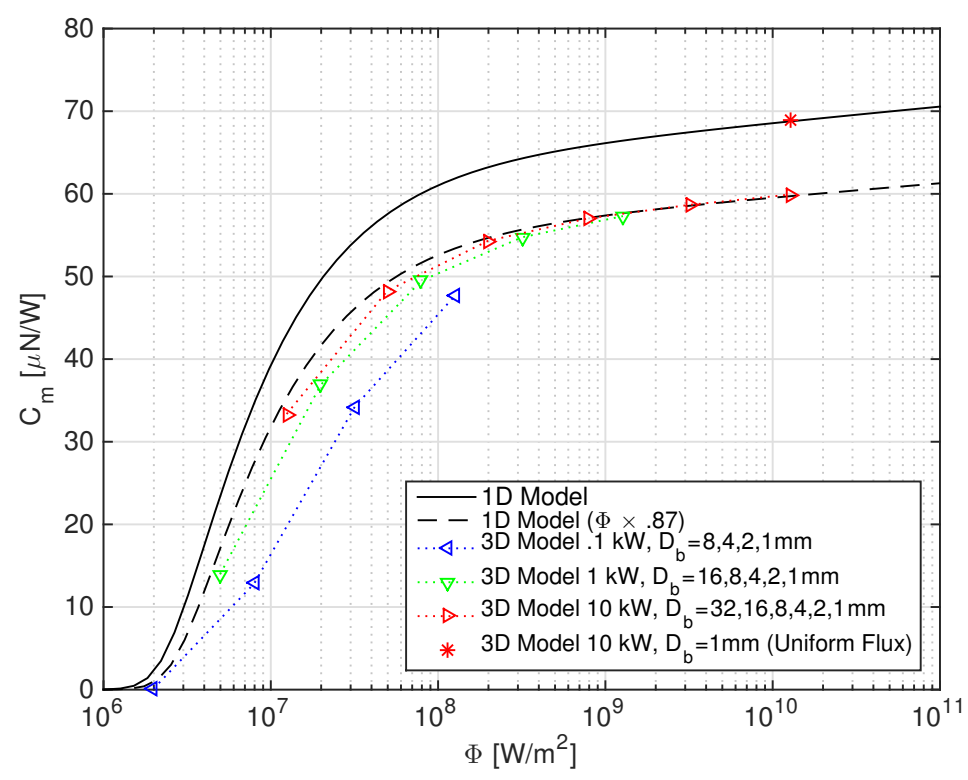

Figure 9: Thrust coupling coefficient as a function of the laser flux

Note that, for a given flux, the $C_{m}$ for the $10 \mathrm{~kW}$ laser case is the closest to the one predicted by the $1 \mathrm{D}$ model. An explanation can be found in the result represented in figure 10 . The figure shows the relative efficiency $\eta$ of the $3 \mathrm{D}$ model as a function of the $D_{b} / l_{c}$ ratio. The relative efficiency $\eta_{3 D}$ is defined as the ratio between the thrust coupling predicted by the $3 \mathrm{D}$ numerical model and the one predicted by the 1D analytical model. Figure 10 shows that when $D_{b}>3 l_{c}, \eta_{3 D}$ is higher than $70 \%$. As the ratio $D_{b} / l_{c}$ increases, $\eta_{3 D}$ tends to an asymptotic value of approximately $87 \%$. The residual $13 \%$ difference between the 3D and 1D predictions is explained by the Gaussian 
intensity distribution of the laser beam. In fact, when a uniform distribution is used instead, $\eta_{3 D}$ tends to $100 \%$ as $D_{b} / l_{c}$ goes to infinity. This can be observed on Figure 9 where the asterisk represents the FEM solution for $10 \mathrm{~kW}$ of optical power and uniform beam distribution.

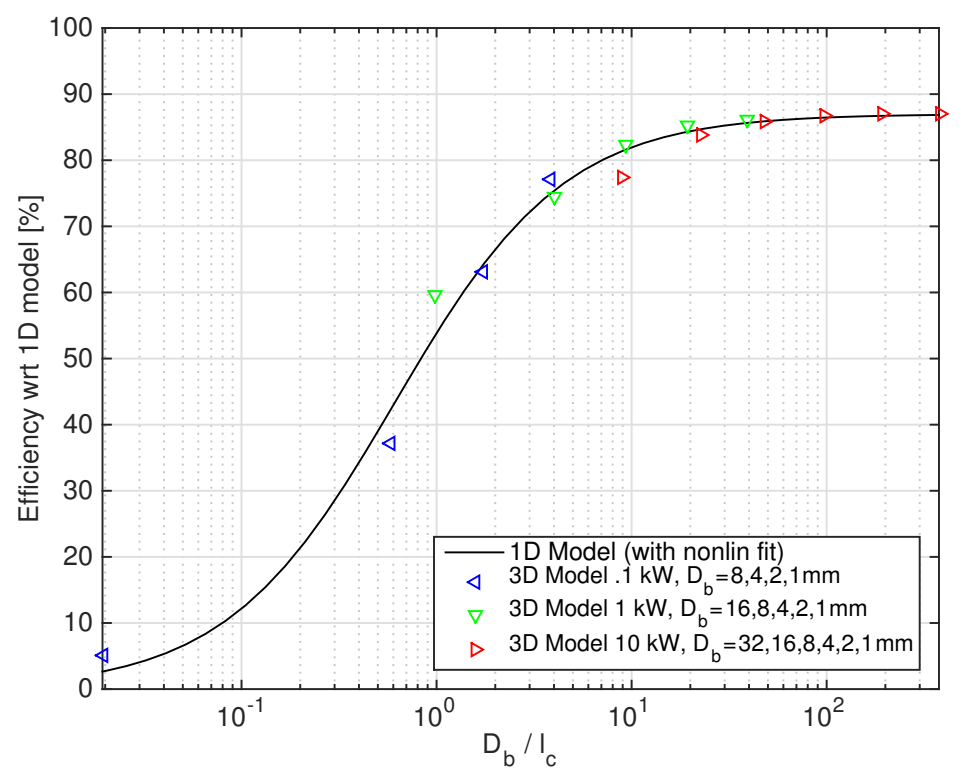

Figure 10: $\eta_{3 D}$ as a function of $\frac{D_{b}}{l_{c}}$

For the case covered in this paper, we found that $\eta_{3 D}$ can be represented, with good accuracy, with the following non-linear fit:

$$
\eta_{3 D}=\frac{1.4136}{1.625+\frac{l_{c}}{D_{b}}}
$$

as shown in Figure 10. If the relative efficiency is applied to the result of the 1D model the $C_{m}$ predicted by the 1D model matches quite well the numerical result (see Figure 9).

\subsection{Effect of Partial Transparency}

Figure 11 shows the temperature distribution under the laser spot for an asteroid made of Forsterite, an output power of $100 \mathrm{~W}$, a beam diameter of 1 millimetre and no partial transparency at the operational wavelength of 

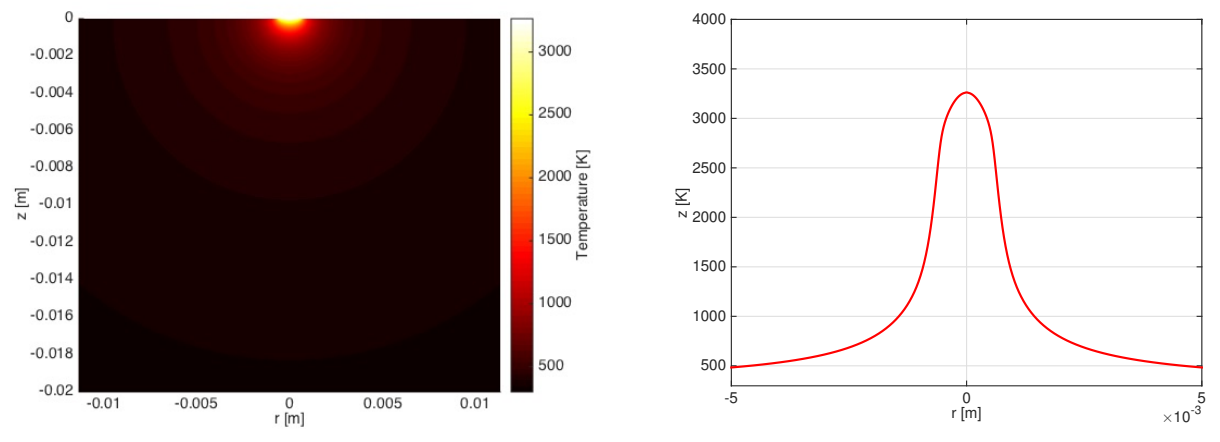

Figure 11: Temperature map inside the material (left) and temperature profile (right) on the upper surface boundary. The optical power is $100 \mathrm{~W}$, the beam diameter is $1 \mathrm{~mm}$ and no partial transparency is considered.

the laser. In this case, the lateral conduction losses are limited and the peak temperature is located at the surface. However, if transparency is considered the absorption length itself can become important in comparison with the size of the laser spot and lateral losses increase significantly. This is due to the fact that the light penetrates deeply in the material and is absorbed by the inner layers. As a result the heat is conducted and radiated sideways and does not contribute to the ablation process. Figure 12 illustrates this phenomenon if an absorption length of $10 \mathrm{~mm}$ is assumed instead. Note that in the case volume absorption is considered, the temperature peak is located under the surface, as explained by Anisimov and Luk'yanchuk (2002). Thiry and Vasile (2014) reported that the correlated color temperature recorded from the light emitted by the ablation spot with a Nd:YAG laser was consistent with the temperature predicted by the model assuming surface absorption. In the remaining, we thus carry on with the assumption that the material is a surface absorber at the laser wavelength.

\section{Losses Due to the Asteroid Motion}

If the target moves under the spot light, the time available to reach the steady-state regime is reduced. With reference to Figure 13, if $v_{r e l}$ is the component of the velocity of a point on the surface in the plane perpendicular to the line of sight of the beam, the mean time of exposure $\tau$ is computed as 

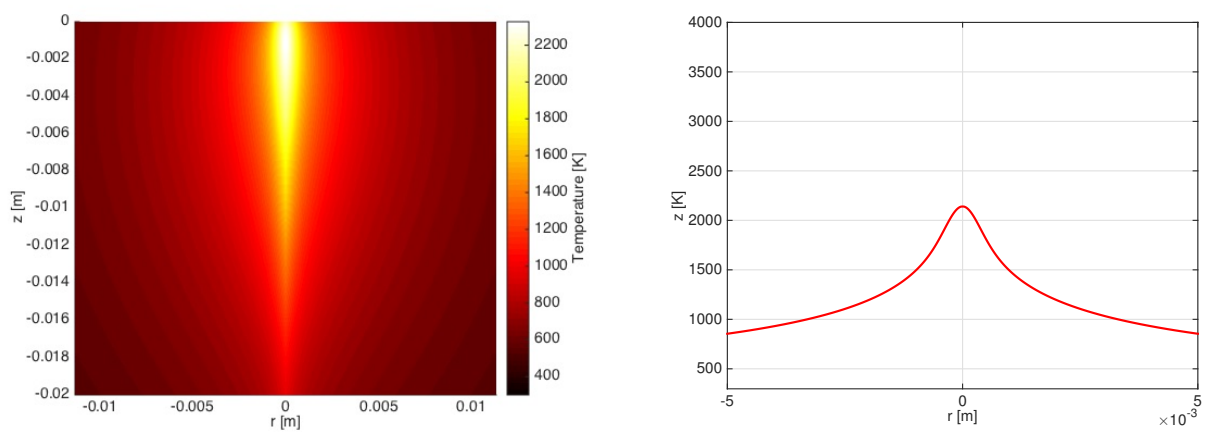

Figure 12: Temperature map inside the material (left) and temperature profile (right) on the upper surface boundary. The optical power is $100 \mathrm{~W}$, the beam diameter is $1 \mathrm{~mm}$ and assumed absorption length of $10 \mathrm{~mm}$.

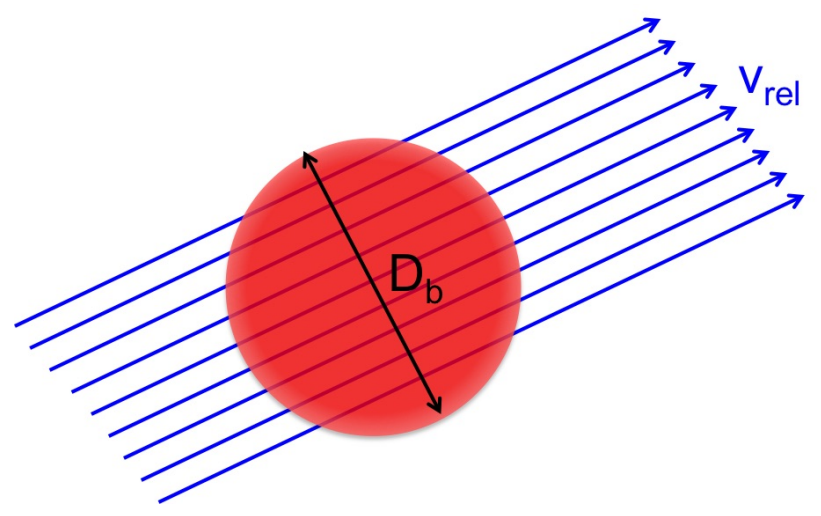

Figure 13: Motion of a surface point under the laser beam

a function of the spot diameter $D_{b}$ as:

$$
\tau=\frac{\pi}{4} \frac{D_{b}}{v_{r e l}}
$$

In the case of a pulsed laser system, this time would simply be the pulse duration and, if this duration is sufficiently short, the coupling coefficient can be made independent from the target motion. Note also that in the general case of an inclined surface, an effective flux must be used in our model by considering the projected area with respect to the angle between the line of sight of the laser beam and the normal to the surface. In order to obtain tractable results, we consider in the rest of this paper the case where 
the illuminated surface is normal to the laser beam even though this is not a limitation in our approach.

\subsection{Finite Volume 1D Transient Model}

The analytical model developed in section 3 is only valid as long as the time available is long compared to the thermal time-scale: $\tau>>\tau_{c}$. When this is not the case, transient effects need to be modelled. The $1 \mathrm{D}$ transient form of the heat equation, using an enthalpy formulation is given by:

$$
\frac{\partial(\rho H)}{\partial t}=-\frac{\partial q}{\partial z}+\frac{\partial\left(\rho u_{v} H\right)}{\partial z}
$$

where the heat flux $q$ is expressed through the common Fourier law $q=-k \frac{d T}{d z}$. As illustrated in Figure 14, Eq. 61) can be discretized by taking $N$ control

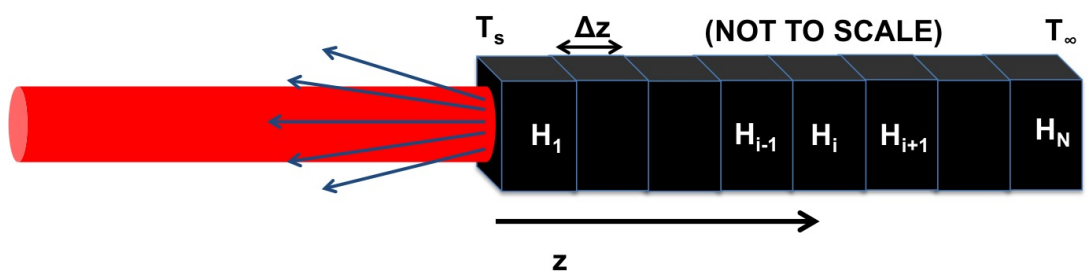

Figure 14: Discretization of the computation domain

volumes along the depth direction $z$ and applying the conservation of the enthalpy $H_{i}$ of each of them as follows:

$$
\frac{d(\rho H)_{i}}{d t}=-\frac{q_{i+1 / 2}-q_{i-1 / 2}}{\Delta z}+u_{v} \frac{(\rho H)_{i+1}-(\rho H)_{i}}{\Delta z}
$$

The fluxes are then computed by taking:

$$
\begin{aligned}
q_{i+1 / 2} & =-k \frac{T_{i+1}-T_{i}}{\Delta z} \\
q_{i-1 / 2} & =-k \frac{T_{i}-T_{i-1}}{\Delta z}
\end{aligned}
$$


The temperature is recovered at each time step from the enthalpy which is for convenience defined equal to 0 at the melting temperature:

$$
\begin{array}{rrr}
T_{i}= & T_{m}+\frac{H_{i}}{c_{s o l}} & \text { if } H_{i} \leq 0 \\
& T_{m} & \text { if } 0<H_{i}<H_{m} \\
& T_{m}+\frac{H_{i}-H_{m}}{c_{l i q}} & \text { if } H_{i} \geq H_{m}
\end{array}
$$

The boundary conditions are then introduced through:

$$
\begin{aligned}
q_{1-1 / 2} & =a \Phi-\epsilon \sigma\left(T_{1}^{4}-T_{\infty}^{4}\right)-\rho u_{v} H_{v} \\
q_{N+1 / 2} & =-k \frac{T_{\infty}-T_{N}}{\Delta z}
\end{aligned}
$$

Eq. (62) is integrated in Matlab ${ }^{\complement}$ using ode23t which is suitable for moderately stiff problems. As an example, Figure 5.1 shows the predicted evolution of the temperature through the depth of the material during 1 millisecond and with a step of $50 \mu \mathrm{s}$ between each curve. As one can see, the temperature distribution quickly progresses towards the exponential distribution.

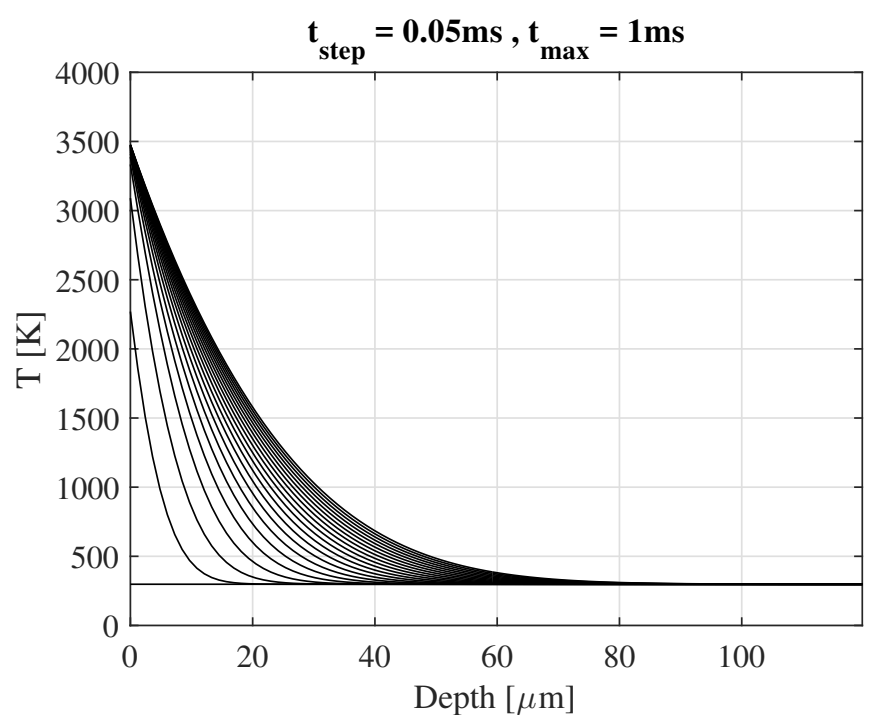

Figure 15: Evolution of the temperature distribution for $\Phi=1 \mathrm{GW} / \mathrm{m}^{2}$ 


\subsection{Effective thrust coupling coefficient and scaled flux}

For a given optical flux, the thrust coupling coefficient $C_{m}$ is now averaged over the total time of exposure $\tau$ :

$$
C_{m}=\frac{\eta_{3 D}}{\tau} \int_{0}^{\tau} C_{m}^{t r}(t) d t
$$

The time-dependent thrust coupling coefficient $C_{m}^{t r}(t)$ is recovered from the surface temperature at each time-step of the computations, using Eq. (23). For a given configuration, the $\eta_{3 D}$ coefficient can be estimated from Eq. (59).

Repeating the calculation for many possible conditions, Figure 16 shows the thrust coupling coefficient as a function of the mean heating time and laser flux. The result in the figure accounts for an assumed $30 \%$ loss due to $3 \mathrm{D}$ effects $\left(\eta_{3 D}=70 \%\right)$. For decreasing heating times, the ablation threshold is shifted towards higher fluxes. In particular, one can see that the asymptotical slope of the isocurves is -2 in logarithmic scales and this validates the fact that the time-scale of the thermal problem varies according to $\Phi^{-2}$, as anticipated in section 3.3 . This observation suggests that the thrust coupling coefficient

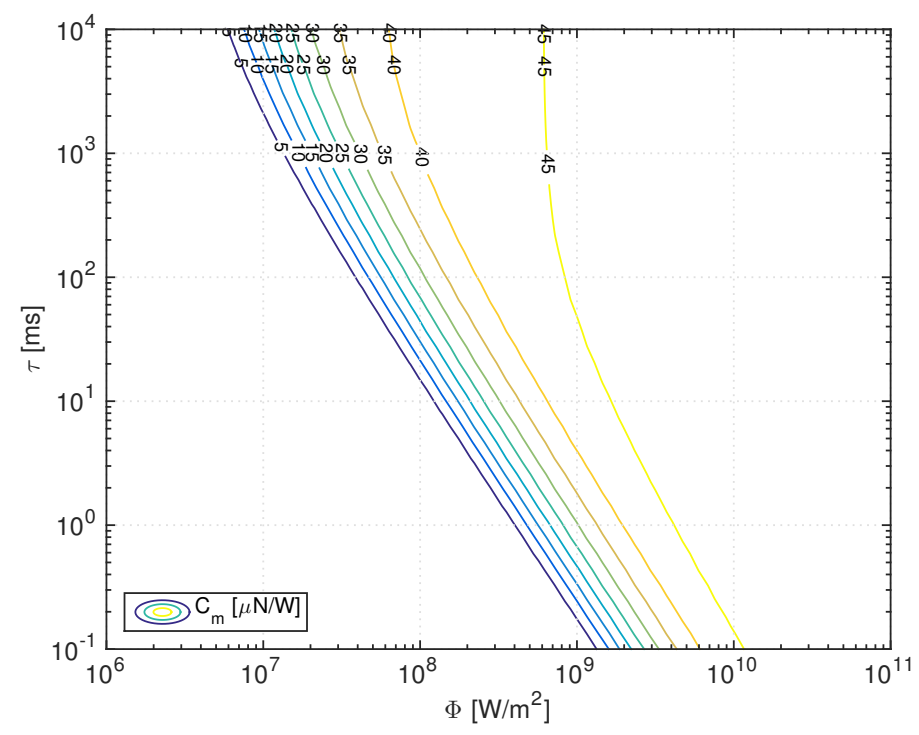

Figure 16: Thrust coupling coefficient as a function of the mean heating time $\tau$ and the optical flux

can be estimated from a universal flux variable $\tilde{\Phi}$ which has the following 
form:

$$
\tilde{\Phi}=\Phi \sqrt{\frac{\tau}{\tau_{0}}}
$$

Assuming again a 30\% loss due to 3D effects, Figure 17 shows the comparison between the Finite Volume Model (FVM) model and the analytical one as a function of the scaled flux variable. By choosing $\tau_{0}$ equal to 10 seconds, one can see that the analytical model is conservative in the range of scaled fluxes that will be considered in the final section of this paper. The FVM curves compare quantitatively well with the predictions of the analytical model. It is worthwhile noting that a somewhat similar result was obtained by Phipps et al. (1988) for the case of pulsed laser systems.

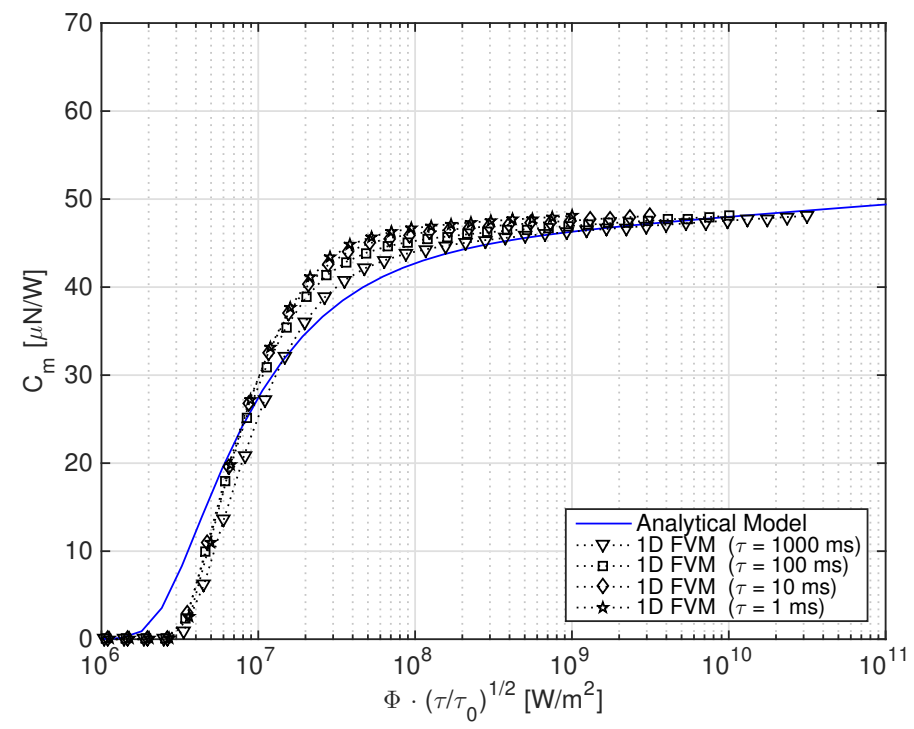

Figure 17: Thrust coupling coefficient in function of the scaled flux $\Phi \sqrt{\frac{\tau}{\tau_{0}}}$ and considering $30 \%$ of losses through $3 \mathrm{D}$ effects

\section{Constraints on the Laser System}

It was shown in section 4 that the different geometrical losses will not exceed $30 \%$ as long as $D_{b}>3 l_{c}$. From section 3.2 , the length $l_{c}$ can be 
approximated as:

$$
l_{c} \approx \frac{k}{a \Phi} \frac{H_{v}}{c}=\frac{\pi}{4} \frac{k D_{b}^{2}}{a P} \frac{H_{v}}{c}
$$

which gives a constraint relating the optical power of the laser and the diameter of the spot:

$$
D_{b}<\frac{4 a c}{3 \pi k H_{v}} P
$$

Furthermore, if one considers the time available to heat a given point on the surface as given by Eq. (60), the scaled flux derived in section 5 can be used to derive a constraint on the optical power of the laser. For example, if one requires a minimum thrust coupling of $35 \mu N$ per Watt of optical power Figure 17 shows that the scaled flux needs to be higher than $20 \mathrm{~W} / \mathrm{mm}^{2}$. Hence, from the definition of the scaled flux, one would get the requirement on the focusing optics:

$$
\frac{4 P}{\pi D_{b}^{2}} \sqrt{\frac{\frac{\pi}{4} \frac{D_{b}}{v_{r e l}}}{\tau_{0}}}>20 \mathrm{E}+06 \Rightarrow D_{b}<6.832 \mathrm{E}-06 \cdot\left(\frac{P^{2}}{v_{r e l}}\right)^{1 / 3}
$$

Note that the constants in this latter expression are only valid for the case of Forsterite. The same analysis carried out in this paper could be repeated for different materials to relate fitting parameters like $\tau_{0}$ to the characterisation of the material properties such as the thermal inertia. A detailed analysis on this topic is however left as the object of future works. Figure 18 shows the minimum optical power required to satisfy both constraints $(73)$ and (74) as a function of the spot diameter for different surface velocities and a minimum thrust of $35 \mu N$ per Watt of optical power. As an example, for a spot diameter of $1 \mathrm{~mm}$ and a surface velocity of $10 \mathrm{~cm} / \mathrm{s}$ a $1 \mathrm{~kW}$ of optical power will be sufficient to generate a continuous thrust of $35 \mathrm{mN}$.

Figure 19 shows the thrust coupling coefficient computed for a surface speed of $10 \mathrm{~cm} / \mathrm{s}$ using the FVM model in combination with the non-linear fit from Eq. 59 accounting for the 3D losses. The white line, with diamond markers, is the minimum power requirement satisfying constraints (73) and (74) ensuring a minimum thrust coupling coefficient of $35 \mu \mathrm{N} / \mathrm{W}$. Note that the actual value is actually closer to $45 \mu \mathrm{N} / \mathrm{W}$ due to the fact that the geometrical losses are in fact closer to $13 \%$ than $30 \%$ for the range of possible combinations between power and beam diameter covered in this specific example. 


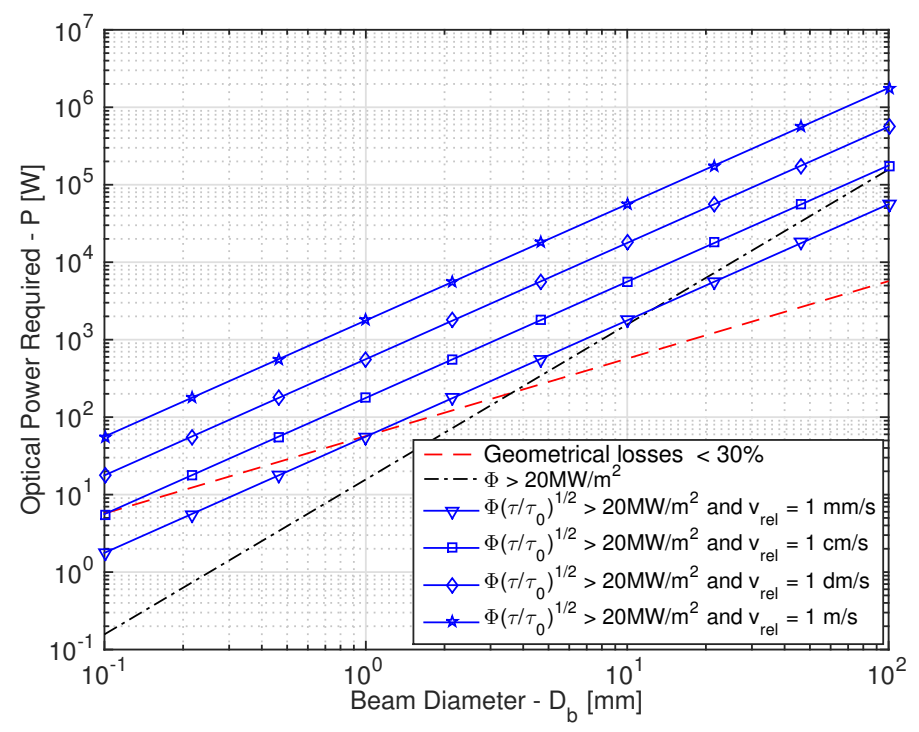

Figure 18: Minimum required optical power as a function of the spot diameter for a $\mathrm{C}_{m} \geq$ $35 \mu \mathrm{N} / \mathrm{W}$

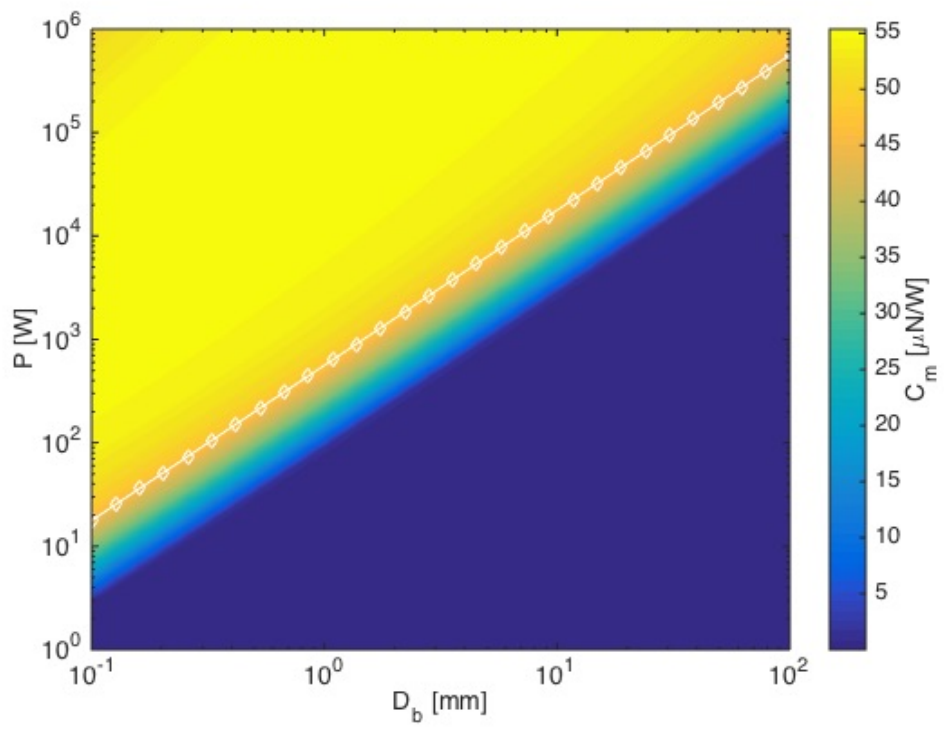

Figure 19: Actual thrust coupling coefficient predicted by the FVM model using the nonlinear fit and considering $\mathrm{v}_{r e l}=10 \mathrm{~cm} / \mathrm{s}$ 


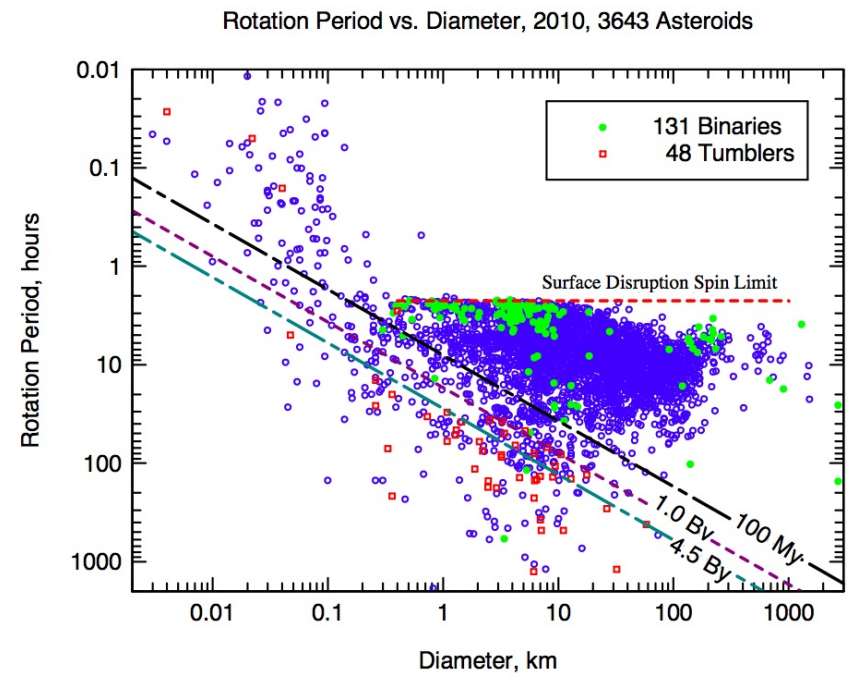

Figure 20: Asteroid Spinning Rate Distribution taken from Sánchez and Scheeres (2014)

In order to put the surface velocity requirement into context, one can look at the asteroid spinning rate distribution in Figure 20. For asteroids larger than $1 \mathrm{~km}$, the spin limit is usually dominated by the gravity stresses and equates

$$
\omega_{\text {lim }}=\sqrt{\frac{4}{3} \pi \rho G} \text { if } D_{\text {ast }}>1 \mathrm{~km}
$$

In a recent paper, Holsapple (2007) proposed a power law for the spin limit of strength-dominated asteroids. With $D_{a s t}$ the diameter of the asteroid in kilometers, the formula that gives a transition at $1 \mathrm{~km}$ is

$$
\omega_{\text {lim }}=D_{\text {ast }}^{-5 / 4} \sqrt{\frac{4}{3} \pi \rho G} \text { if } D_{\text {ast }} \leqslant 1 \mathrm{~km}
$$

For spherical asteroids ${ }^{6}$ of a given size, the maximum surface velocity $v_{r e l}$ can thus be computed as:

$$
v_{r e l} \leqslant \omega_{\text {lim }} \frac{D_{a s t}}{2}
$$

\footnotetext{
${ }^{6}$ Note that this expression can easily be extended to the general case by taking into account the elongation factor of the shape
} 
Assuming again a $C_{m}$ of $35 \mu N$ per Watt of optical power, the required optical power to achieve a given deflection objective can then be computed. For instance the yearly rate of velocity change $\Delta v_{y r}$ is equal to

$$
\Delta v_{y r}=365.242 \cdot 86400 \frac{6 C_{m} P_{o p t}}{\pi \rho D_{a s t}^{3}}
$$

The required power as a function of the velocity change per year and asteroid size can be seen on Figure 21. The required focusing ability of the optics can be seen on Figure 22 as a function of the required optical power and asteroid size.

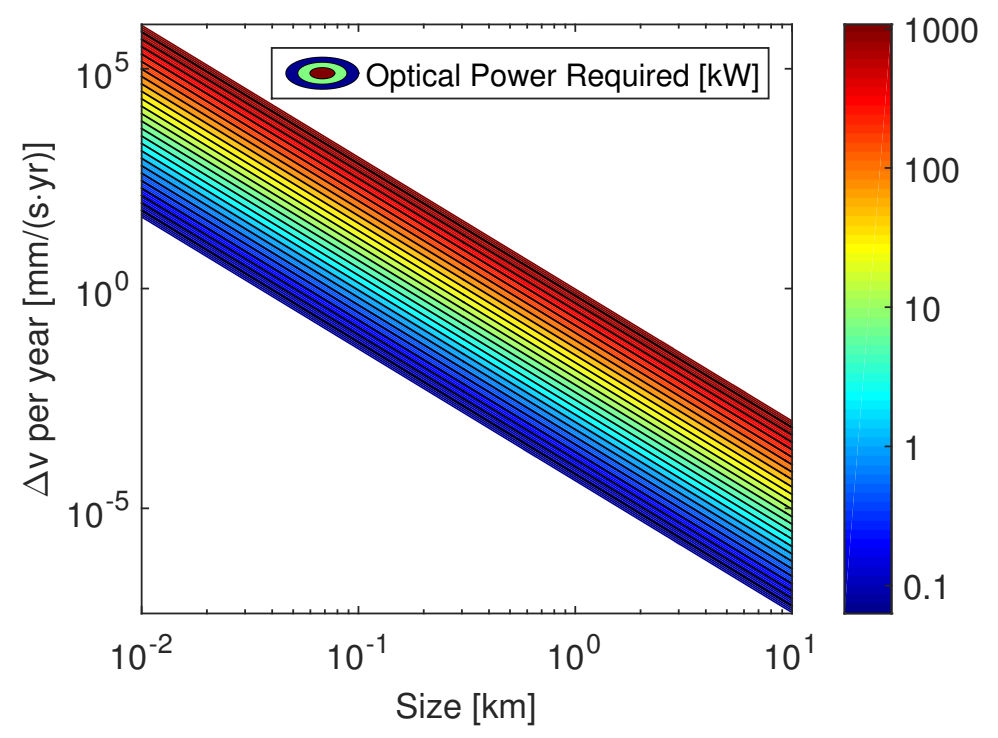

Figure 21: Optical power required as a function of the asteroid size and $\Delta v$ per year

As a final note, the size of the optics will scale with the inverse of the required spot diameter. If $D_{\text {primary }}$ is the diameter of the primary mirror, $l_{f}$ the focal length of the focusing optics, the diffraction limit imposes that the minimum mirror diameter is:

$$
D_{\text {primary }}=\mathcal{M}^{2} \frac{4 \lambda l_{f}}{\pi D_{b}}
$$

In which $\mathcal{M}^{2}$ is a beam quality factor accounting for the higher order modes (=1 for a purely Gaussian beam). Considering a shooting distance of $1 \mathrm{~km}$, 


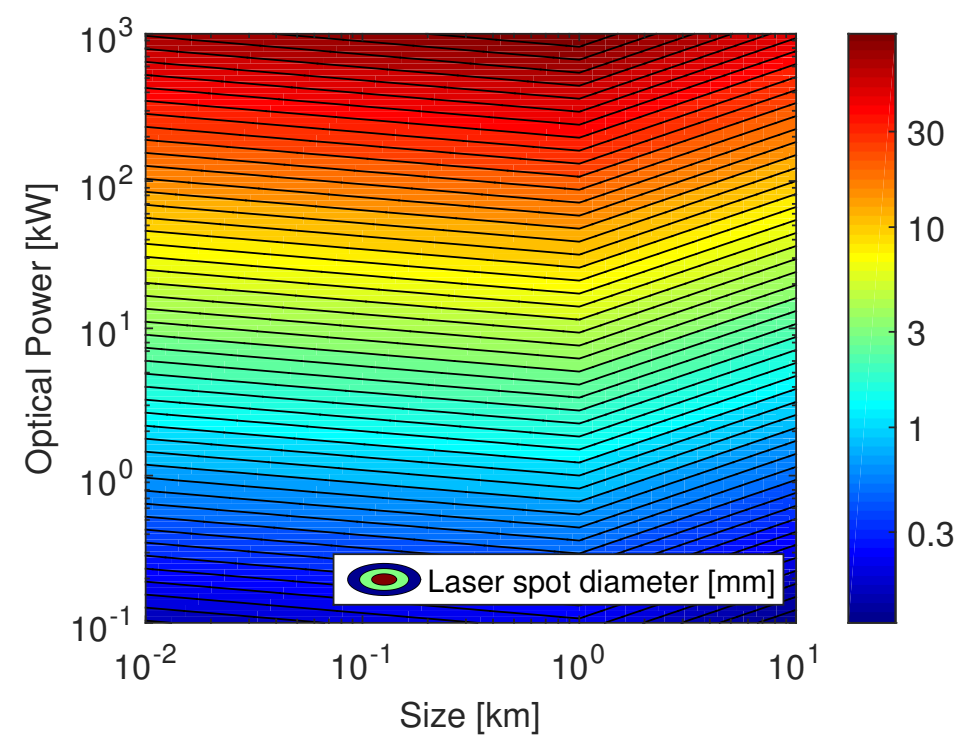

Figure 22: spot size to achieve at the focal distance to guarantee the performance

a laser wavelength of $1.06 \mu m$, and a poor quality factor of 3, spot sizes comprised between $0.1 \mathrm{~mm}$ and $100 \mathrm{~mm}$ would require a primary mirror ranging from $40.5 \mathrm{~m}$ down to $4 \mathrm{~cm}$. For very large powers, a fractionated solution would however be preferred in order to reduce the thermal stresses on the optical components. This topic is left as the object of a future study.

\section{Conclusion}

This paper presented a straightforward approach to estimate the thrust coupling due to laser ablation completed with a thorough analysis of the losses due to 3D thermal conduction and the movement of the free surface illuminated by the laser. By comparing the results against a 3D simulation, we showed that lateral conduction losses are negligible as long as the thickness of the heated layer is small compared to the size of the laser beam. Through dimensional analysis and a transient simulation, we also showed the existence of a flux-dependent time-scale to the thermal problem, which must be sufficiently small compared to the time available to heat the surface for a significant thrust to be generated. Using correction laws, the analytical model was demonstrated to give results that are consistent with respect to the numerical models and accurate enough to allow sizing the laser system 
in order to deliver the required thrust. Our main conclusion is that the focusing optics required to achieve a minimum thrust coupling value of $35 \mu \mathrm{N}$ per Watt of optical power depends on the available laser power and relative motion of the illuminated surface. To illustrate these results, we estimated the required power and focusing ability of the onboard laser system in order to achieve a given deflection objective for a wide range of asteroid sizes.

\section{Acknowledgement}

This work is funded by the European Commission's Framework Programme 7, through the Stardust Marie Curie Initial Training Network, FP7PEOPLE-2012-ITN, Grant Agreement 317185.

\section{References}

Ahrens, T. J., O'Keefe, J. D., 1972. Shock melting and vaporization of lunar rocks and minerals. The Moon 4 (1-2), 214-249.

Anisimov, S. I., Khokhlov, V. A., 1995. Instabilities in laser-matter interaction. CRC press.

Anisimov, S. I., Luk'yanchuk, B., 2002. Selected problems of laser ablation theory. Physics-Uspekhi 45 (3), 293-324.

Brashears, T., Lubin, P., Hughes, G. B., Meinhold, P., Suen, J., Batliner, P., Motta, C., Griswold, J., Kangas, M., Johansson, I., et al., 2015. Directed energy deflection laboratory measurements. In: SPIE Optical Engineering + Applications. International Society for Optics and Photonics.

Choudhury, N., Chaplot, S., Rao, K., 1989. Equation of state and melting point studies of forsterite. Physics and Chemistry of Minerals 16 (6), 599605 .

Gibbings, A., Vasile, M., Watson, I., Hopkins, J.-M., Burns, D., 2013. Experimental analysis of laser ablated plumes for asteroid deflection and exploitation. Acta Astronautica 90 (1), 85 - 97, NEO Planetary Defense: From Threat to Action.

Holsapple, K. A., 2007. Spin limits of solar system bodies: From the small fast-rotators to 2003 el61. Icarus 187 (2), 500-509. 
Kahle, R., Kührt, E., Hahn, G., Knollenberg, J., 2006. Physical limits of solar collectors in deflecting Earth-threatening asteroids. Aerospace Science and Technology 10, 253-263.

Knight, C. J., 1979. Theoretical modeling of rapid surface vaporization with back pressure. AIAA journal 17 (5), 519-523.

Park, S.-Y., Mazanek, D. D., Jan-Mar 2005. Deflection of Earth-crossing asteroids/comets using rendezvous spacecraft and laser ablation. Journal of Astronautical Sciences 53 (1), 21-37.

Phipps, C., Albrecht, G., Friedman, H., Gavel, D., George, E., Murray, J., Ho, C., Priedhorsky, W., Michaelis, M., Reilly, J., 1996. Orion: Clearing near-earth space debris using a 20-kw, 530-nm, earth-based, repetitively pulsed laser. Laser and Particle Beams 14 (01), 1-44.

Phipps, C., Turner, T., Harrison, R., York, G., Osborne, W., Anderson, G., Corlis, X., Haynes, L., Steele, H., Spicochi, K., et al., 1988. Impulse coupling to targets in vacuum by krf, hf, and co2 single-pulse lasers. Journal of Applied Physics 64 (3), 1083-1096.

Poueyo-Verwaerde, A., Fabbro, R., Deshors, G., De Frutos, A., Orza, J., 1993. Experimental study of laser-induced plasma in welding conditions with continuous co2 laser. Journal of applied physics 74 (9), 5773-5780.

Sánchez, P., Scheeres, D. J., 2014. The strength of regolith and rubble pile asteroids. Meteoritics \& Planetary Science 49 (5), 788-811.

Thiry, N., Vasile, M., 2014. Recent advances in laser ablation modelling for asteroid deflection methods. In: SPIE Optical Engineering + Applications. International Society for Optics and Photonics.

Vasile, M., Gibbings, A., Massimo, V., Sanchez, J.-P., Yarnoz, D., Eckersley, S., Wayman, A., Branco, J., Burns, D., Hopkins, J.-M., Colombo, C., McInnes, C., 2013. Light touch2: Effective solutions to asteroid manipulation. sysnova challenge analysis final report. Tech. rep., University of Strathclyde.

Vasile, M., Gibbings, A., Watson, I., Hopkins, J.-M., 2014. Improved laser ablation model for asteroid deflection. Acta Astronautica 103, 382-394. 
Vasile, M., Maddock, C., May 2010. On the deflection of asteroids with mirrors. Celestial Mechanics and Dynamical Astronomy 107 (1), 265-284.

Vasile, M., Maddock, C. A., 2012. Design of a formation of solar pumped lasers for asteroid deflection. Advances in Space Research 50 (7), 891-905.

Vertes, A., De Wolf, M., Juhasz, P., Gijbels, R., 1989. Threshold conditions of plasma ignition in laser ionization mass spectrometry of solids. Analytical Chemistry 61 (9), 1029-1035.

Vertes, A., Gijbels, R., Adams, F., 1993. Laser ionization mass analysis. Wiley New York.

Yoo, S.-M., Songa, Y.-J., Park, S.-Y., Choi, K.-H., 2009. Spacecraft formation flying for Earth-crossing object deflections using a power limited laser ablating. Advances in Space Research 43 (12), 1873-1889.

Zuiani, F., Vasile, M., Gibbings, A., 2012. Evidence-based robust design of deflection actions for near earth objects celestial mechanics and dynamical astronomy. Celestial Mechanics and Dynamical Astronomy 114 (1-2), 107136. 